\title{
POISSON VERTEX ALGEBRA COHOMOLOGY AND DIFFERENTIAL HARRISON COHOMOLOGY
}

\author{
BOJKO BAKALOV, ALBERTO DE SOLE, VICTOR G. KAC, AND VERONICA VIGNOLI
}

To Nikolai Reshetikhin on his 60-th birthday.

\begin{abstract}
We construct a canonical map from the Poisson vertex algebra cohomology complex to the differential Harrison cohomology complex, which restricts to an isomorphism on the top degree. This is an important step in the computation of Poisson vertex algebra and vertex algebra cohomologies.
\end{abstract}

\section{INTRODUCTION}

The present paper is a next step in the development of the cohomology theory of vertex algebras started in [BDSHK18, BDSHK19]. Recall (see e.g. [BDSHK18]) that, to any linear symmetric (super)operad $\mathcal{P}$ over a field $\mathbb{F}$, one canonically associates a $\mathbb{Z}$-graded Lie superalgebra

$$
W(\mathcal{P})=\bigoplus_{k=-1}^{\infty} W^{k}(\mathcal{P}), \quad \text { where } \quad W^{k}(\mathcal{P})=\mathcal{P}(k+1)^{S_{k+1}}
$$

An odd element $X \in W^{1}(\mathcal{P})$, satisfying $[X, X]=0$, defines a cohomology complex $(W(\mathcal{P}), \operatorname{ad} X)$, which is a differential graded Lie superalgebra.

A cohomology theory of vertex algebras is constructed by considering the operad $\mathcal{P}_{\text {ch }}(V)$, attached to a vector superspace $V$ with an even endomorphism $\partial$. In order to describe this construction, let, for $n \in \mathbb{Z}_{\geq 0}$,

$$
V_{n}=V\left[\lambda_{1}, \ldots, \lambda_{n}\right] /\left\langle\partial+\lambda_{1}+\cdots+\lambda_{n}\right\rangle,
$$

where the indeterminates $\lambda_{i}$ have even parity and $\langle\Phi\rangle$ stands for the image of the endomorphism $\Phi$, and let

$$
\mathcal{O}_{n}^{\star, T}=\mathbb{F}\left[z_{i}-z_{j},\left(z_{i}-z_{j}\right)^{-1}\right]_{1 \leq i<j \leq n} .
$$

The superspace $\mathcal{P}_{\mathrm{ch}}(V)(n)$ is defined as the set of all linear maps

$$
Y: V^{\otimes n} \otimes \mathcal{O}_{n}^{\star, T} \rightarrow V_{n}, \quad v_{1} \otimes \cdots \otimes v_{n} \otimes f \mapsto Y_{\lambda_{1}, \ldots, \lambda_{n}}\left(v_{1} \otimes \cdots \otimes v_{n} \otimes f\right),
$$

satisfying the following two sesquilinearity properties $(1 \leq i \leq n)$ :

$$
Y_{\lambda_{1}, \ldots, \lambda_{n}}\left(v_{1} \otimes \cdots \otimes\left(\partial+\lambda_{i}\right) v_{i} \otimes \cdots \otimes v_{n} \otimes f\right)=Y_{\lambda_{1}, \ldots, \lambda_{n}}\left(v_{1} \otimes \cdots \otimes v_{n} \otimes \frac{\partial f}{\partial z_{i}}\right),
$$

and

$$
Y_{\lambda_{1}, \ldots, \lambda_{n}}\left(v_{1} \otimes \cdots \otimes v_{n} \otimes\left(z_{i}-z_{j}\right) f\right)=\left(\frac{\partial}{\partial \lambda_{j}}-\frac{\partial}{\partial \lambda_{i}}\right) Y_{\lambda_{1}, \ldots, \lambda_{n}}\left(v_{1} \otimes \cdots \otimes v_{n} \otimes f\right) .
$$

Key words and phrases. Poisson vertex algebra, Harrison cohomology, chiral operad, classical operad, Poisson vertex algebra cohomology, variational Poisson cohomology. 
In [BDSHK18] we also defined the action of $S_{n}$ on $\mathcal{P}_{\mathrm{ch}}(V)(n)$ and the $\circ_{i}$-products, making $\mathcal{P}_{\text {ch }}(V)$ an operad.

As a result, we obtain the Lie superalgebra

$$
W_{\mathrm{ch}}(V)=W\left(\mathcal{P}_{\mathrm{ch}}(V)\right)=\bigoplus_{k=-1}^{\infty} W_{\mathrm{ch}}^{k}(V),
$$

see (1.1). We show in [BDSHK18] that odd elements $X \in W_{\mathrm{ch}}^{1}(\Pi V)$, such that $[X, X]=0$, correspond bijectively to vertex algebra structures on the $\mathbb{F}[\partial]$-module $V$, such that $\partial$ is the translation operator (where $\Pi$ stands for the reversal of parity). This leads to the vertex algebra cohomology complex $\left(W_{\mathrm{ch}}(\Pi V)\right.$, ad $\left.X\right)$, with coefficients in the adjoint $V$-module. The cohomology with coefficients in an arbitrary $V$-module $M$ in obtained by a simple reduction procedure.

Now, suppose that the $\mathbb{F}[\partial]$-module $V$ has an increasing $\mathbb{Z}_{\geq 0}$-filtration by $\mathbb{F}[\partial]$ submodules. Taking the increasing filtration of $\mathcal{O}_{n}^{\star}, T$ by the number of divisors, we obtain an increasing filtration of $V^{\otimes n} \otimes \mathcal{O}_{n}^{\star, T}$. This filtration induces a decreasing filtration of the superspace $\mathcal{P}_{\mathrm{ch}}(V)(n)$ (see [BDSHK18]). The associated graded spaces gr $\mathcal{P}_{\mathrm{ch}}(V)(n)$ form a graded operad.

On the other hand, in [BDSHK18] we introduced the closely related operad $\mathcal{P}_{\mathrm{cl}}(V)$, which "governs" the Poisson vertex algebra (PVA) structures on the $\mathbb{F}[\partial]$ module $V$. Let $\mathbb{F} \mathcal{G}(n)$ be the vector space (with even parity) spanned by the set $\mathcal{G}(n)$ of labeled oriented graphs with $n$ vertices. The vector superspace $\mathcal{P}_{\mathrm{cl}}(V)(n)$ is the space of linear maps (cf. (1.2))

$$
Y: \mathbb{F} \mathcal{G}(n) \otimes V^{\otimes n} \rightarrow V_{n}, \quad \Gamma \otimes v \mapsto Y^{\Gamma}(v),
$$

satisfying the sesquilinearity conditions (3.28) and (3.29) in Section 3.7, which are the "classical" analogs of (1.3) and (1.4). The corresponding $\mathbb{Z}$-graded Lie superalgebra

$$
W_{\mathrm{cl}}(\Pi V)=\bigoplus_{k=-1}^{\infty} W_{\mathrm{cl}}^{k}(\Pi V)
$$

is such that odd elements $X \in W_{\mathrm{cl}}^{1}(\Pi V)$ with $[X, X]=0$ parametrize the PVA structures on the $\mathbb{F}[\partial]$-module $V$ by

$$
a b=(-1)^{p(a)} X^{\bullet \rightarrow \bullet}(a \otimes b), \quad\left[a_{\lambda} b\right]=(-1)^{p(a)} X_{\lambda,-\lambda-\partial}^{\bullet}(a \otimes b) .
$$

When $V$ is endowed with an increasing $\mathbb{Z}_{\geq 0}$-filtration by $\mathbb{F}[\partial]$-submodules, we have a canonical linear map of graded operads

$$
\operatorname{gr} \mathcal{P}_{\mathrm{ch}}(V) \rightarrow \mathcal{P}_{\mathrm{cl}}(\operatorname{gr} V) .
$$

It is proved in [BDSHK18] that the map (1.7) is injective. The main result of [BDSHK19] is that this map is an isomorphism, provided that the filtration of $V$ is induced by a grading by $\mathbb{F}[\partial]$-modules. If, in addition, this filtration of $V$ is such that gr $V$ inherits from the vertex algebra structure of $V$ a PVA structure, then, as a result, the vertex algebra cohomology is majorized by the classical PVA cohomology:

$$
\operatorname{dim} H_{\mathrm{ch}}^{n}(V) \leq \operatorname{dim} H_{\mathrm{cl}}^{n}(V) .
$$

Unfortunately, (1.8) is not a "practical" inequality, since the direct computation of $H_{\mathrm{cl}}(V, M)$ may be very hard. However, if the superspace $V$ is endowed with 
the structure of a commutative associative superalgebra with an even derivation $\partial$, there exists a much smaller $\mathbb{Z}$-graded Lie superalgebra, constructed in [DSK13]:

$$
W_{\mathrm{PV}}(\Pi V)=\bigoplus_{k=-1}^{\infty} W_{\mathrm{PV}}^{k}(\Pi V) .
$$

It has the property that PVA structures on the differential algebra $V$ correspond bijectively to odd elements $X \in W_{\mathrm{PV}}^{1}(\Pi V)$ such that $[X, X]=0$, cf. (1.6). This produces the variational Poisson cohomology complex $\left(W_{\mathrm{PV}}(\Pi V)\right.$, ad $\left.X\right)$. It is easy to see that this complex is a subcomplex of the complex $\left(W_{\mathrm{cl}}(\Pi V)\right.$, ad $\left.X\right)$, corresponding to the graphs without edges.

The present paper is the first paper towards proving that the inclusion of complexes

$$
\left(W_{\mathrm{PV}}(\Pi V), \operatorname{ad} X\right) \hookrightarrow\left(W_{\mathrm{cl}}(\Pi V), \operatorname{ad} X\right),
$$

induces an isomorphism in cohomology, under some assumptions on the differential algebra $V$. In this case, we may replace $H_{\mathrm{cl}}$ by $H_{\mathrm{PV}}$ in (1.8). Since there are by now well developed tools for computing variational Poisson cohomology, see [DSK13] and [BDSK19], this allows one to get a hold of the vertex algebra cohomology.

In the present paper, given a vector superspace $V$ with an even endomorphism $\partial$, we construct a canonical map of complexes

$$
\left(W_{\mathrm{cl}}(\Pi V), \operatorname{ad} X\right) \stackrel{\varphi}{\longrightarrow}\left(C_{\partial, \mathrm{Har}}(V), d\right),
$$

which restricts to a bijective linear map on the top degree:

$$
\mathrm{gr}^{n-1} W_{\mathrm{cl}}^{n-1}(\Pi V) \stackrel{\sim}{\longrightarrow} C_{\partial, \mathrm{Har}}^{n}(V),
$$

see Theorem 4.1 in Section 4 . Here $X \in W_{\mathrm{cl}}^{1}(\Pi V)$ corresponds to the PVA structure on $V$, given by (1.6). In particular, $V$ is endowed with a structure of a commutative associative superalgebra with an even derivation $\partial$, hence we may consider the differential Harrison complex

$$
\left(C_{\partial, \mathrm{Har}}(V)=\bigoplus_{n \in \mathbb{Z}_{\geq 0}} C_{\partial, \mathrm{Har}}^{n}(V), d\right)
$$

Here $C_{\partial, \operatorname{Har}}^{n}(V)$ is the subspace of $\operatorname{Hom}_{\mathbb{F}[\partial]}\left(V^{\otimes n}, V\right)$ satisfying Harrison's conditions (2.11) in Section 2.3. It was shown in [Har62, GS87] that the subspace $C_{\partial, \operatorname{Har}}(V)$ of $\bigoplus_{n \in \mathbb{Z}_{\geq 0}} \operatorname{Hom}_{\mathbb{F}[\partial]}\left(V^{\otimes n}, V\right)$ is invariant with respect to the Hochschild differential $d$ (which commutes with $\partial$ ). Finally, the map $\varphi$ in (1.10) maps $Y \in W_{\mathrm{cl}}^{n-1}(\Pi V)$ to $Y^{\Lambda_{n}}$, where $\Lambda_{n}$ is the labeled oriented graph

$$
\Lambda_{n}=\underset{12}{\longrightarrow} \cdots \underset{n}{\longrightarrow}
$$

In our next paper [BDSHKV19] we will show that if the differential superalgebra $V$ is such that $H_{\partial, \operatorname{Har}}^{n}(V, d)=0$ for $n>1$, then the inclusion (1.10) is a quasiisomorphism, hence in this case the inequality (1.8) turns into the inequality

$$
\operatorname{dim} H_{\mathrm{ch}}^{n}(V) \leq \operatorname{dim} H_{\mathrm{PV}}^{n}(V) .
$$

We refer to the Ph.D. thesis [Vig19] for examples and more details.

Throughout the paper, the base field $\mathbb{F}$ has characteristic 0 , and, unless otherwise specified, all vector spaces, their tensor products and Hom's are over $\mathbb{F}$. 
Acknowledgments. This research was partially conducted during the authors' visits to the University of Rome La Sapienza, to MIT, and to IHES. The first author was supported in part by a Simons Foundation grant 584741. The second author was partially supported by the national PRIN fund n. 2015ZWST2C_001 and the University funds n. RM116154CB35DFD3 and RM11715C7FB74D63. The third author was partially supported by the Bert and Ann Kostant fund and by a Simons Fellowship.

\section{Differential Harrison Cohomology COMPlex}

In this section, we recall the definition of the Harrison cohomology complex and we introduce the differential Harrison cohomology complex.

2.1. Hochschild cohomology complex. First, we review the Hochschild cohomology complex, of which Harrison's is a subcomplex, see [Hoc45] and [Har62]. We use the original Harrison's definition. For other definitions see [GS87, Lod13].

Let $A$ be an associative algebra over the base field $\mathbb{F}$, and $M$ be an $A$-bimodule. We will write $A^{\otimes n}$ for the $n$-fold tensor product $A \otimes \cdots \otimes A$. The Hochschild cohomology complex is defined as follows. The space of $n$-cochains is

$$
\operatorname{Hom}\left(A^{\otimes n}, M\right),
$$

and the differential $d: \operatorname{Hom}\left(A^{\otimes n}, M\right) \rightarrow \operatorname{Hom}\left(A^{\otimes n+1}, M\right)$ is defined by

$$
\begin{aligned}
(d f)\left(a_{1} \otimes\right. & \left.\cdots \otimes a_{n+1}\right)=a_{1} f\left(a_{2} \otimes \cdots \otimes a_{n+1}\right) \\
& +\sum_{i=1}^{n}(-1)^{i} f\left(a_{1} \otimes \cdots \otimes a_{i-1} \otimes a_{i} a_{i+1} \otimes a_{i+2} \otimes \cdots \otimes a_{n+1}\right) \\
& +(-1)^{n+1} f\left(a_{1} \otimes \cdots \otimes a_{n}\right) a_{n+1} .
\end{aligned}
$$

Then $d^{2}=0$, and we get the Hochschild cohomology complex

$$
0 \longrightarrow M \stackrel{d}{\longrightarrow} \operatorname{Hom}(A, M) \stackrel{d}{\longrightarrow} \operatorname{Hom}\left(A^{\otimes 2}, M\right) \stackrel{d}{\longrightarrow} \cdots .
$$

If $A$ is an associative algebra with a derivation $\partial: A \rightarrow A$, and $M$ is a differential bimodule over $A$ (i.e., the action of $\partial$ is compatible with the bimodule structure), we may consider the differential Hochschild cohomology complex by taking the subspace of $n$-cochains

$$
\operatorname{Hom}_{\mathbb{F}[\partial]}\left(A^{\otimes n}, M\right) .
$$

It is clear by the definition (2.2) that the differential $d$ maps $\operatorname{Hom}_{\mathbb{F}[\partial]}\left(A^{\otimes n}, M\right)$ to $\operatorname{Hom}_{\mathbb{F}[\partial]}\left(A^{\otimes n+1}, M\right)$. Hence, we have a cohomology subcomplex.

Remark 2.1. It is straightforward, using the Koszul-Quillen rule, to extend the definition of the Hochschild complex to the case when $A$ is an associative superalgebra, as well as all other definitions and results of the paper. We restricted here to the purely even case for the simplicity of the exposition.

2.2. Monotone permutations. Consider the symmetric group $S_{n}$. Using Harrison's notation in [Har62] (see also [GS87]), we have the following definition:

Definition 2.2. A permutation $\pi \in S_{n}$ is called monotone if, for each $i=1, \ldots, n$, one of the following two conditions holds:

(a) $\pi(j)<\pi(i)$ for all $j<i$;

(b) $\pi(j)>\pi(i)$ for all $j<i$. 
(Not necessarily the same condition (a) or (b) holds for every $i$.) When (b) holds, we call $i$ a drop of $\pi$. Also, $\pi(1)=k$ is called the start of $\pi$ (and we say that $\pi$ starts at $k$ ).

We denote by $\mathcal{M}_{n} \subset S_{n}$ the set of monotone permutations, and by $\mathcal{M}_{n}^{k} \subset \mathcal{M}_{n}$ the set of monotone permutations starting at $k$.

Here is a simple description of all monotone permutations starting at $k$. Let us identify the permutation $\pi \in S_{n}$ with the $n$-tuple $[\pi(1), \ldots, \pi(n)]$. To construct all $\pi \in \mathcal{M}_{n}^{k}$, we let $\pi(1)=k$. Then, for every choice of $k-1$ positions in $\{2, \ldots, n\}$ we get a monotone permutation $\pi$ as follows. In the selected positions we put the numbers 1 to $k-1$ in decreasing order from left to right; in the remaining positions we write the numbers $k+1$ to $n$ in increasing order from left to right. (The selected positions are the drops of $\pi$.)

According to the above description, we have a bijective correspondence

$$
\mathcal{M}_{n}^{k} \stackrel{\sim}{\rightarrow}\{D \subset\{2, \ldots, n\}|| D \mid=k-1\},
$$

associating the monotone permutation $\pi \in \mathcal{M}_{n}^{k}$ to the set $D(\pi)$ of drops of $\pi$, which are

$$
\pi^{-1}(k-1)<\pi^{-1}(k-2)<\cdots<\pi^{-1}(1) \in\{2, \ldots, n\} .
$$

Example 2.3. The only monotone permutation starting at 1 is the identity, while the only monotone permutation starting at $n$ is

$$
\sigma_{n}=\left[\begin{array}{lllll}
n & n-1 & \cdots & 2 & 1
\end{array}\right] .
$$

Example 2.4. Let $n=5$ and $k=3$. The monotone permutations starting at 3 are

$$
\begin{aligned}
& {\left[\begin{array}{lllll}
3 & \underline{2} & 1 & 4 & 5
\end{array}\right], \quad\left[\begin{array}{lllll}
3 & \underline{2} & 4 & \underline{1} & 5
\end{array}\right],\left[\begin{array}{lllll}
3 & \underline{2} & 4 & 5 & 1
\end{array}\right],} \\
& {\left[\begin{array}{lllll}
3 & 4 & \underline{2} & 1 & 5
\end{array}\right], \quad\left[\begin{array}{lllll}
3 & 4 & 2 & 5 & 1
\end{array}\right],\left[\begin{array}{lllll}
3 & 4 & 5 & \underline{2} & 1
\end{array}\right] \text {, }}
\end{aligned}
$$

where we underlined the positions of the drops.

Given a monotone permutation $\pi$, we denote by $\operatorname{dr}(\pi)$ the sum of all the drops with respect to $\pi$. According to the previous description, we can easily see that

$$
(-1)^{\operatorname{dr}(\pi)}=(-1)^{k-1} \operatorname{sign}(\pi),
$$

if $k$ is the start of $\pi$.

Note that the description (2.5) of $\mathcal{M}_{n}^{k}$ in terms of positions of drops allows us to count the number of elements in $\mathcal{M}_{n}^{k}$, for fixed $n$ and $k$. We have:

$$
\left|\mathcal{M}_{n}^{k}\right|=\left(\begin{array}{l}
n-1 \\
k-1
\end{array}\right) .
$$

Remark 2.5. Let us denote by $\mathcal{M}_{n}^{k, k-1} \subset \mathcal{M}_{n}^{k}$ the subset of all monotone permutations $\pi$ starting at $k$ with $\pi(2)=k-1$, and by $\mathcal{M}_{n}^{k, k+1} \subset \mathcal{M}_{n}^{k}$ the subset of all monotone permutations $\pi$ starting at $k$ with $\pi(2)=k+1$. Then we have

$$
\mathcal{M}_{n}^{k}=\mathcal{M}_{n}^{k, k-1} \sqcup \mathcal{M}_{n}^{k, k+1} .
$$

Lemma 2.6. There are natural identifications

$$
\mathcal{M}_{n}^{k, k-1} \simeq \mathcal{M}_{n-1}^{k-1} \quad\left(\text { resp. } \mathcal{M}_{n}^{k, k+1} \simeq \mathcal{M}_{n-1}^{k}\right),
$$


mapping $\pi \in \mathcal{M}_{n}^{k, k-1}$ to $\bar{\pi} \in \mathcal{M}_{n-1}^{k-1}$ (resp. $\pi \in \mathcal{M}_{n}^{k, k+1}$ to $\bar{\pi} \in \mathcal{M}_{n-1}^{k}$ ), given by $\bar{\pi}(1):=k-1$ (resp. $k$ ), and, for $i=2, \ldots, n-1$,

$$
\bar{\pi}(i):=\left\{\begin{array}{ll}
\pi(i+1), & \text { if } \pi(i+1)<k \\
\pi(i+1)-1, & \text { if } \quad \pi(i+1)>k
\end{array} .\right.
$$

Moreover,

$$
(-1)^{\mathrm{dr}(\bar{\pi})}=(-1)^{\mathrm{dr}(\pi)+k} \quad\left(\text { resp. }(-1)^{\mathrm{dr}(\pi)+k-1}\right) .
$$

Proof. Straightforward; see [Vig19] for details.

Remark 2.7. Observe that, given a monotone permutation $\pi$, either $\pi(n)=1$ or $\pi(n)=n$. Denote by ${ }^{1} \mathcal{M}_{n}^{k} \subset \mathcal{M}_{n}^{k}$ the set of all the monotone permutations $\pi$ starting at $k$ with $\pi(n)=1$, and by ${ }^{n} \mathcal{M}_{n}^{k} \subset \mathcal{M}_{n}^{k}$ the set of all the monotone permutations $\pi$ starting at $k$ with $\pi(n)=n$. As in Remark 2.5, we have

$$
\mathcal{M}_{n}^{k}={ }^{1} \mathcal{M}_{n}^{k} \sqcup^{n} \mathcal{M}_{n}^{k}
$$

Lemma 2.8. There are natural identifications

$$
{ }^{1} \mathcal{M}_{n}^{k} \simeq \mathcal{M}_{n-1}^{k-1} \quad\left(\text { resp. }{ }^{n} \mathcal{M}_{n}^{k} \simeq \mathcal{M}_{n-1}^{k}\right),
$$

mapping $\pi \in{ }^{1} \mathcal{M}_{n}^{k}$ to $\tilde{\pi} \in \mathcal{M}_{n-1}^{k-1}$ (resp. $\pi \in{ }^{n} \mathcal{M}_{n}^{k}$ to $\tilde{\pi} \in \mathcal{M}_{n-1}^{k}$ ), given by $\tilde{\pi}(i):=\pi(i)-1$ (resp. $\tilde{\pi}(i):=\pi(i))$, for $i=1, \ldots, n-1$. Moreover,

$$
(-1)^{\mathrm{dr}(\tilde{\pi})}=(-1)^{\mathrm{dr}(\pi)+n} \quad\left(\text { resp. }(-1)^{\mathrm{dr}(\pi)}\right)
$$

Proof. Straightforward; see [Vig19] for details.

2.3. Differential Harrison cohomology complex. Let us now recall Harrison's original definition of his cohomology complex [Har62]. Let $A$ be a commutative associative algebra, and $M$ be a symmetric $A$-bimodule, i.e., such that $a m=m a$, for all $a \in A$ and $m \in M$. For every $1<k \leq n$ define the following endomorphism on the space $\operatorname{Hom}\left(A^{\otimes n}, M\right)$ :

$$
\left(L_{k} F\right)\left(a_{1} \otimes \cdots \otimes a_{n}\right):=\sum_{\pi \in \mathcal{M}_{n}^{k}}(-1)^{\mathrm{dr}(\pi)} F\left(a_{\pi(1)} \otimes \cdots \otimes a_{\pi(n)}\right) .
$$

A Harrison $n$-cochain is defined as a Hochschild $n$-cochain $F \in \operatorname{Hom}\left(A^{\otimes n}, M\right)$ fixed by all operators $L_{k}$ :

$$
L_{k} F=F, \text { for every } 2 \leq k \leq n
$$

We will denote by

$$
C_{\mathrm{Har}}^{n}(A, M) \subset \operatorname{Hom}\left(A^{\otimes n}, M\right)
$$

the space of Harrison $n$-cochains.

Furthermore, if $A$ is a differential algebra with a derivation $\partial: A \rightarrow A$, and $M$ is a symmetric differential bimodule, we may consider the space of differential Harrison $n$-cochains

$$
C_{\partial, \operatorname{Har}}^{n}(A, M) \subset \operatorname{Hom}_{\mathbb{F}[\partial]}\left(A^{\otimes n}, M\right),
$$

again defined by Harrison's conditions (2.11).

Proposition 2.9. (a) The Harrison complex $\left(C_{\mathrm{Har}}(A, M), d\right)$ is a subcomplex of the Hochschild complex.

(b) If $A$ is a differential algebra, with a derivation $\partial: A \rightarrow A$, the differential Harrison complex $\left(C_{\partial, \mathrm{Har}}(A, M), d\right)$ is a subcomplex of the differential Hochschild complex. 
Proof. The proof of (a) is in [Har62, GS87]. Part (b) is straightforward.

Remark 2.10. Clearly, $H_{\partial, \mathrm{Har}}^{0}(A, M)=M$ and $H_{\partial, \mathrm{Har}}^{1}(A, M)=\operatorname{Der}_{\mathbb{F}[\partial]}(A, M)$. It follows from [GS87] that $H_{\partial \text {,Har }}^{n}(A, M)$ is a direct summand of the differential Hochschild cohomology $H H_{\partial}^{n}(A, M)$, for $n \geq 2$.

\section{The Classical operad AND PVA COHOMOLOGY}

In this section, we recall some basic notions that will be used throughout the paper, and review the construction of the PVA cohomology complex as described in [BDSHK18].

3.1. Symmetric group actions. There is a natural left action of $S_{n}$ on an arbitrary $n$-tuple of objects $\left(\lambda_{1}, \ldots, \lambda_{n}\right)$ :

$$
\sigma\left(\lambda_{1}, \ldots, \lambda_{n}\right)=\left(\lambda_{\sigma^{-1}(1)}, \ldots, \lambda_{\sigma^{-1}(n)}\right), \quad \sigma \in S_{n} .
$$

Also, given $V=V_{\overline{0}} \oplus V_{\overline{1}}$ a vector superspace with parity $p$, we have a linear left action of the symmetric group $S_{n}$ on the tensor product $V^{\otimes n}\left(\sigma \in S_{n}, v_{1}, \ldots, v_{n} \in V\right)$ :

$$
\sigma\left(v_{1} \otimes \cdots \otimes v_{n}\right):=\epsilon_{v}(\sigma) v_{\sigma^{-1}(1)} \otimes \cdots \otimes v_{\sigma^{-1}(n)},
$$

where, following the Koszul-Quillen rule,

$$
\epsilon_{v}(\sigma):=\prod_{i<j: \sigma(i)>\sigma(j)}(-1)^{p\left(v_{i}\right) p\left(v_{j}\right)} .
$$

In particular, if $V$ is purely even $\epsilon_{v}(\sigma)=1$, while if $V$ is purely odd $\epsilon_{v}(\sigma)=\operatorname{sign}(\sigma)$. The corresponding right action of $S_{n}$ on the the space $\operatorname{Hom}\left(V^{\otimes n}, V\right)$ is given by $\left(f \in \operatorname{Hom}\left(V^{\otimes n}, V\right), \sigma \in S_{n}\right)$ :

$$
f^{\sigma}\left(v_{1} \otimes \cdots \otimes v_{n}\right)=f\left(\sigma\left(v_{1} \otimes \cdots \otimes v_{n}\right)\right) .
$$

3.2. Composition of permutations and shuffles. Let $n \geq 1$ and $m_{1}, \ldots, m_{n} \geq$ 0 . We introduce the following notation:

$$
M_{0}=0 \text { and } M_{i}=\sum_{j=1}^{i} m_{j}, \quad i=1, \ldots, n .
$$

Given $\sigma \in S_{n}$ and $\tau_{1} \in S_{m_{1}}, \ldots, \tau_{n} \in S_{m_{n}}$, we describe the composition

$$
\sigma\left(\tau_{1}, \ldots, \tau_{n}\right) \in S_{M_{n}}
$$

by saying how it acts on the tensor power $V^{\otimes M_{n}}$ of a vector space $V$ :

$\left(\sigma\left(\tau_{1}, \ldots, \tau_{n}\right)\right)\left(v_{1} \otimes \cdots \otimes v_{M_{n}}\right)=\sigma\left(\tau_{1}\left(v_{1} \otimes \cdots \otimes v_{M_{1}}\right) \otimes \cdots \otimes \tau_{n}\left(v_{M_{n-1}+1} \otimes \cdots \otimes v_{M_{n}}\right)\right)$.

Definition 3.1. A permutation $\sigma \in S_{m+n}$ is called an $(m, n)$-shuffle if

$$
\sigma(1)<\cdots<\sigma(m), \quad \sigma(m+1)<\cdots<\sigma(m+n) .
$$

The subset of $(m, n)$-shuffles is denoted by $S_{m, n} \subset S_{m+n}$.

Observe that, by definition, $S_{0, n}=S_{n, 0}=\{1\}$ for every $n \geq 0$. If either $m$ or $n$ is negative, we set $S_{m, n}=\emptyset$ by convention. 
3.3. $n$-graphs. For an oriented graph $\Gamma$, we denoted by $V(\Gamma)$ the set of vertices of $\Gamma$, and by $E(\Gamma)$ the set of edges. We call $\Gamma$ an $n$-graph if $V(\Gamma)=\{1, \ldots, n\}$. Denote by $\mathcal{G}(n)$ the set of all $n$-graphs without tadpoles, and by $\mathcal{G}_{0}(n)$ the set of all acyclic $n$-graphs.

An $n$-graph $L$ will be called an $n$-line, or simply a line, if its set of edges is of the form $\left\{i_{1} \rightarrow i_{2}, i_{2} \rightarrow i_{3}, \ldots, i_{n-1} \rightarrow i_{n}\right\}$, where $\left\{i_{1}, \ldots, i_{n}\right\}$ is a permutation of $\{1, \ldots, n\}$.

We have a natural left action of $S_{n}$ on the set $\mathcal{G}(n)$ : for the $n$-graph $\Gamma$ and the permutation $\sigma$, the new $n$-graph $\sigma(\Gamma)$ is defined to be the same graph as $\Gamma$ but with the vertex which was labeled as $i$ relabeled as $\sigma(i)$, for every $i=1, \ldots, n$. So, if the $n$-graph $\Gamma$ has an oriented edge $i \rightarrow j$, then the $n$-graph $\sigma(\Gamma)$ has the oriented edge $\sigma(i) \rightarrow \sigma(j)$. Note that $S_{n}$ permutes the set of $n$-lines.

Let us recall the cocomposition of $n$-graphs, as described in [BDSHK18]. Given an $n$-tuple $\left(m_{1}, \ldots, m_{n}\right)$ of positive integers, let $M_{i}$ be as in (3.5). If $\Gamma \in \mathcal{G}\left(M_{n}\right)$, define $\Delta_{i}^{m_{1}, \ldots, m_{n}}(\Gamma) \in \mathcal{G}\left(m_{i}\right), i=1, \ldots, n$, to be the subgraph of $\Gamma$ associated to the set of vertices $\left\{M_{i-1}+1, \ldots, M_{i}\right\}$, relabeled as $\left\{1, \ldots, m_{i}\right\}$. Define also $\Delta_{0}^{m_{1}, \ldots, m_{n}}(\Gamma)$ to be the graph obtained from $\Gamma$ by collapsing the vertices and the edges of each $\Delta_{i}^{m_{1}, \ldots, m_{n}}(\Gamma)$ into a single vertex, relabeled as $i$. Then the cocomposition map is the map

$$
\begin{aligned}
\Delta^{m_{1}, \ldots, m_{n}}: \mathcal{G}\left(M_{n}\right) & \rightarrow \mathcal{G}(n) \times \mathcal{G}\left(m_{1}\right) \times \cdots \times \mathcal{G}\left(m_{n}\right), \\
\Gamma & \mapsto\left(\Delta_{0}^{m_{1}, \ldots, m_{n}}(\Gamma), \Delta_{1}^{m_{1}, \ldots, m_{n}}(\Gamma), \ldots, \Delta_{n}^{m_{1}, \ldots, m_{n}}(\Gamma)\right) .
\end{aligned}
$$

Example 3.2. Let $n=3,\left(m_{1}, m_{2}, m_{3}\right)=(3,1,4)$, and $\Gamma \in \mathcal{G}(8)$ be the following graph

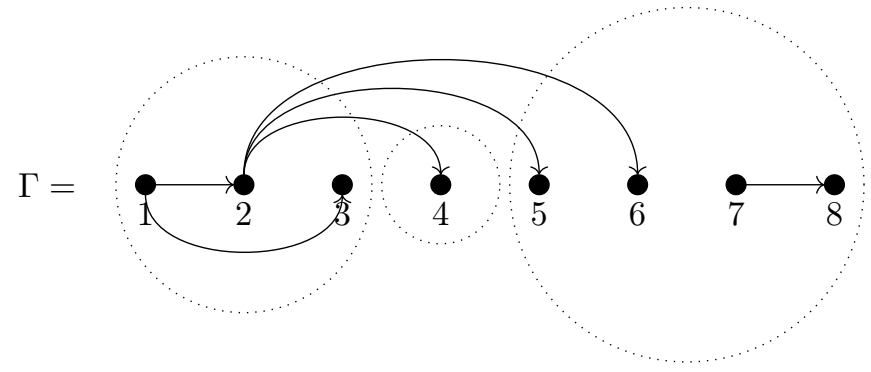

The cocomposition $\Delta^{3,1,4}(\Gamma)=\left(\Delta_{0}^{3,1,4}(\Gamma), \Delta_{1}^{3,1,4}(\Gamma), \Delta_{2}^{3,1,4}(\Gamma), \Delta_{3}^{3,1,4}(\Gamma)\right)$ is given by the following graphs. $\Delta_{1}^{3,1,4}(\Gamma)$ is the subgraph of $\Gamma$ generated by the first three vertices:

$$
\Delta_{1}^{3,1,4}(\Gamma)=\underset{2}{\mathbf{8}} \underset{3}{\mathbf{8}} \in \mathcal{G}(3)
$$

$\Delta_{2}^{3,1,4}(\Gamma)$ is the subgraph of $\Gamma$ associated to the fourth vertex:

$$
\Delta_{2}^{3,1,4}(\Gamma)=\underset{1}{\bullet} \in \mathcal{G}(1)
$$

$\Delta_{3}^{3,1,4}(\Gamma)$ is the subgraph of $\Gamma$ associated to the last four vertices:

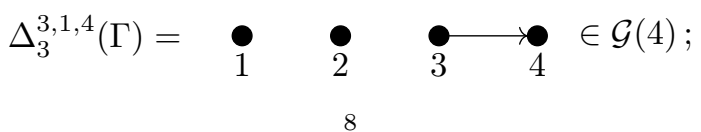


and, finally, $\Delta_{0}^{3,1,4}(\Gamma)$ is:

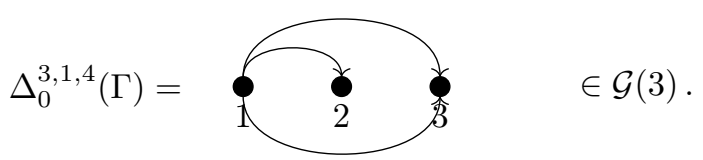

From the construction of $\Delta_{i}^{m_{1}, \ldots, m_{n}}(\Gamma)$, it is easy to see that there is a natural bijective correspondence

$$
\Delta: E(\Gamma) \stackrel{\sim}{\longrightarrow} E\left(\Delta_{0}^{m_{1} \ldots m_{n}}(\Gamma)\right) \sqcup E\left(\Delta_{1}^{m_{1} \ldots m_{n}}(\Gamma)\right) \sqcup \cdots \sqcup E\left(\Delta_{n}^{m_{1} \ldots m_{n}}(\Gamma)\right) .
$$

Definition 3.3. Let $k \in\left\{1, \ldots, M_{n}\right\}$ and $j \in\{1, \ldots, n\}$. We say that $j$ is externally connected to $k$ (via the graph $\Gamma$ and its cocomposition $\Delta^{m_{1} \ldots m_{n}}(\Gamma)$ ) if there is an unoriented path (without repeating edges) of $\Delta_{0}^{m_{1} \ldots m_{n}}(\Gamma)$ joining $j$ to $i$, where $i \in\{1, \ldots, n\}$ is such that $k \in\left\{M_{i-1}+1, \ldots, M_{i}\right\}$, and the edge out of $i$ is the image, via the map $\Delta$ in (3.9), of an edge which has its head or tail in $k$. Given a set of variables $x_{1}, \ldots, x_{n}$, we denote

$$
X(k)=\sum_{\begin{array}{c}
j \text { externally } \\
\text { connected to } k
\end{array}} x_{j} .
$$

Example 3.4. For the graph (3.8), we have

$X(1)=0, X(2)=x_{1}+x_{2}+x_{3}, X(3)=0, X(4)=x_{1}+x_{3}, X(5)=x_{1}+x_{2}+x_{3}$, $X(6)=x_{1}+x_{2}+x_{3}, X(7)=0, X(8)=0$.

\subsection{Lie conformal algebras and Poisson vertex algebras.}

Definition 3.5. A Lie conformal (super)algebra is a vector (super)space $V$, endowed with an even endomorphism $\partial \in \operatorname{End}(V)$ and a bilinear (over $\mathbb{F}$ ) $\lambda$-bracket $\left[\cdot{ }_{\lambda} \cdot\right]: V \times V \rightarrow V[\lambda]$ satisfying sesquilinearity $(a, b \in V)$ :

$$
\left[\partial a_{\lambda} b\right]=-\lambda\left[a_{\lambda} b\right], \quad\left[a_{\lambda} \partial b\right]=(\lambda+\partial)\left[a_{\lambda} b\right],
$$

skewsymmetry $(a, b \in V)$ :

$$
\left[a_{\lambda} b\right]=-(-1)^{p(a) p(b)}\left[b_{-\lambda-\partial} a\right],
$$

and the Jacobi identity $(a, b, c \in V)$ :

$$
\left[a_{\lambda}\left[b_{\mu} c\right]\right]-(-1)^{p(a) p(b)}\left[b_{\mu}\left[a_{\lambda}, b\right]\right]=\left[\left[a_{\lambda} b\right]_{\lambda+\mu} c\right] .
$$

Definition 3.6. A Poisson vertex (super)algebra (PVA) is a commutative associative (super)algebra $V$ endowed with an even derivation $\partial$ and a Lie conformal (super)algebra $\lambda$-bracket $\left[\cdot \lambda^{\cdot} \cdot\right]$ that satisfies the left Leibniz rule

$$
\left[a_{\lambda} b c\right]=\left[a_{\lambda} b\right] c+(-1)^{p(a) p(b)} b\left[a_{\lambda} c\right] .
$$

3.5. Operads. Recall that a (linear, unital, symmetric) superoperad $\mathcal{P}$ is a collection of vector superspaces $\mathcal{P}(n), n \geq 0$, with parity $p$, endowed, for every $f \in \mathcal{P}(n)$ and $m_{1}, \ldots, m_{n} \geq 0$, with a composition parity preserving linear map,

$$
\begin{aligned}
\mathcal{P}(n) \otimes \mathcal{P}\left(m_{1}\right) \otimes \cdots \otimes \mathcal{P}\left(m_{n}\right) & \rightarrow \mathcal{P}\left(M_{n}\right), \\
f \otimes g_{1} \otimes \cdots \otimes g_{n} & \mapsto f\left(g_{1} \otimes \cdots \otimes g_{n}\right),
\end{aligned}
$$


where $M_{n}$ is as in (3.5), satisfying the following associativity axiom:

$$
f\left(\left(g_{1} \otimes \cdots \otimes g_{n}\right)\left(h_{1} \otimes \cdots \otimes h_{M_{n}}\right)\right)=\left(f\left(g_{1} \otimes \cdots \otimes g_{n}\right)\right)\left(h_{1} \otimes \cdots \otimes h_{M_{n}}\right) \in \mathcal{P}\left(\sum_{j=1}^{M_{n}} \ell_{j}\right),
$$

for every $f \in \mathcal{P}(n), g_{i} \in \mathcal{P}\left(m_{i}\right)$ for $i=1, \ldots, n$, and $h_{j} \in \mathcal{P}\left(\ell_{j}\right)$ for $j=1, \ldots, M_{n}$. In the left-hand side of (3.16) the linear map

$$
g_{1} \otimes \cdots \otimes g_{n}: \bigotimes_{j=1}^{M_{n}} \mathcal{P}\left(\ell_{j}\right) \rightarrow \bigotimes_{i=1}^{n} \mathcal{P}\left(\sum_{j=M_{i-1}+1}^{M_{i}} \ell_{j}\right)
$$

is the tensor product of composition maps applied to

$h_{1} \otimes \cdots \otimes h_{M_{n}}=\left(h_{1} \otimes \cdots \otimes h_{M_{1}}\right) \otimes\left(h_{M_{1}+1} \otimes \cdots \otimes h_{M_{2}}\right) \otimes \cdots \otimes\left(h_{M_{n-1}+1} \otimes \cdots \otimes h_{M_{n}}\right)$.

We assume that $\mathcal{P}$ is endowed with a unit element $1 \in \mathcal{P}(1)$ satisfying the following unity axioms:

$$
f(1 \otimes \cdots \otimes 1)=1(f)=f, \text { for every } f \in \mathcal{P}(n) .
$$

Furthermore, we assume that, for each $n \geq 1, \mathcal{P}(n)$ has a right action of the symmetric group $S_{n}$, denoted $f^{\sigma}$, for $f \in \mathcal{P}(n)$ and $\sigma \in S_{n}$, satisfying the following equivariance axiom $\left(f \in \mathcal{P}(n), g_{1} \in \mathcal{P}\left(m_{1}\right), \ldots, g_{n} \in \mathcal{P}\left(m_{n}\right), \sigma \in S_{n}\right.$, $\left.\tau_{1} \in S_{m_{1}}, \ldots, \tau_{n} \in S_{m_{n}}\right)$ :

$$
f^{\sigma}\left(g_{1}^{\tau_{1}} \otimes \cdots \otimes g_{n}^{\tau_{n}}\right)=\left(f\left(\sigma\left(g_{1} \otimes \cdots \otimes g_{n}\right)\right)\right)^{\sigma\left(\tau_{1}, \ldots, \tau_{n}\right)},
$$

where the left action of $\sigma \in S_{n}$ on the tensor product of vector superspaces was defined in (3.2), and the composition $\sigma\left(\tau_{1}, \ldots, \tau_{n}\right)$ is described in (3.6).

For simplicity, from now on, we will use the term operad in place of superoperad. Given an operad $\mathcal{P}$, one defines, for each $i=1, \ldots, n$, the $\circ_{i}$-product $\circ_{i}: \mathcal{P}(n) \otimes$ $\mathcal{P}(m) \rightarrow \mathcal{P}(n+m-1)$ by insertion in position $i$, i.e.

$$
f \circ_{i} g=f(\overbrace{1 \otimes \cdots \otimes 1}^{i-1} \otimes g \otimes \overbrace{1 \otimes \cdots \otimes 1}^{i}) .
$$

Example 3.7. The simplest example of an operad is $\mathcal{P}=\mathcal{H}$ om. Given a vector superspace $V, \mathcal{H o m}=\mathcal{H o m}(V)$ is defined as the collection of

$$
\mathcal{H o m}(n):=\operatorname{Hom}\left(V^{\otimes n}, V\right), \quad n \geq 0,
$$

endowed with the composition maps $\left(f \in \mathcal{H o m}(n), g_{i} \in \mathcal{H o m}\left(m_{i}\right)\right.$ for $i=1, \ldots, n$, $v_{j} \in V$ for $j=1, \ldots, M_{n}$ )

$$
\left(f\left(g_{1} \otimes \cdots \otimes g_{n}\right)\right)\left(v_{1} \otimes \cdots \otimes v_{M_{n}}\right):=f\left(\left(g_{1} \otimes \cdots \otimes g_{n}\right)\left(v_{1} \otimes \cdots \otimes v_{M_{n}}\right)\right),
$$

where $M_{n}$ is as in (3.5). Hom is a unital operad with unity $1=\mathbb{1}_{V} \in \operatorname{End}(V)$, and the right action of $S_{n}$ on $\mathcal{H o m}(n)$ is given by (3.4).

3.6. The $\mathbb{Z}$-graded Lie superalgebra associated to an operad. Recall that, given an operad $\mathcal{P}$, one can construct the associated $\mathbb{Z}$-graded Lie superalgebra $W(\mathcal{P})$. It is defined, as a $\mathbb{Z}$-graded vector superspace

$$
W(\mathcal{P})=\sum_{n \geq-1} W^{n}(\mathcal{P})=\sum_{n \geq-1} \mathcal{P}(n+1)^{S_{n+1}}
$$


with the following Lie bracket. For $f \in W^{n}(\mathcal{P})$ and $g \in W^{m}(\mathcal{P})$, their $\square$-product is defined by

$$
f \square g=\sum_{\sigma \in S_{m+1, n}}\left(f \circ_{1} g\right)^{\sigma^{-1}} \in W^{m+n}(\mathcal{P})
$$

and the Lie bracket on $W(\mathcal{P})$ is given by

$$
[f, g]=f \square g-(-1)^{p(f) p(g)} g \square f .
$$

See e.g. [BDSHK18, Sec. 3] for details.

3.7. The classical operad $\mathcal{P}_{\mathrm{cl}}$ [BDSHK18]. Let $V=V_{\overline{0}} \oplus V_{\overline{1}}$ be a vector superspace with parity $p$, endowed with an even endomorphism $\partial \in \operatorname{End} V$. For $n \geq 0$, define $\mathcal{P}_{\mathrm{cl}}(n)$ as the vector superspace of all maps

$$
f: \mathcal{G}(n) \times V^{\otimes n} \longrightarrow V\left[\lambda_{1}, \ldots, \lambda_{n}\right] /\left\langle\partial+\lambda_{1}+\cdots+\lambda_{n}\right\rangle,
$$

which are linear in the second factor, mapping the $n$-graph $\Gamma \in \mathcal{G}(n)$ (by definition $p(\Gamma)=\overline{0})$ and the monomial $v_{1} \otimes \cdots \otimes v_{n} \in V^{\otimes n}$ to the polynomial

$$
f_{\lambda_{1}, \ldots, \lambda_{n}}^{\Gamma}\left(v_{1} \otimes \cdots \otimes v_{n}\right),
$$

satisfying the cycle relations and the sesquilinearity conditions described as follows.

The cycle relations say that

$$
\text { if } \Gamma \notin \mathcal{G}_{0}(n), \text { then } f^{\Gamma}=0,
$$

and

$$
\text { if } C \subset E(\Gamma) \text { is an oriented cycle of } \Gamma, \text { then } \sum_{e \in C} f^{\Gamma \backslash e}=0,
$$

where $\Gamma \backslash e$ is the graph obtained from $\Gamma$ by removing the edge $e$. Observe that for oriented cycles of length 2 , the cycle relation (3.26) means that changing orientation of a single edge of the $n$-graph $\Gamma \in \mathcal{G}(n)$ amounts to a change of sign of $f^{\Gamma}$.

The sesquilinearity conditions are as follows. Let $\Gamma=\Gamma_{1} \sqcup \cdots \sqcup \Gamma_{s}$ be the decomposition of $\Gamma$ as a disjoint union of its connected components, and let $I_{1}, \ldots, I_{s} \subset$ $\{1, \ldots, n\}$ be the sets of vertices associated to these connected components. For a graph $\tilde{\Gamma}$ and its set of vertices $\tilde{I} \subset\{1, \ldots, n\}$, we write

$$
\lambda_{\tilde{\Gamma}}=\sum_{i \in \tilde{I}} \lambda_{i}, \quad \partial_{\tilde{\Gamma}}=\sum_{i \in \tilde{I}} \partial_{i},
$$

where $\partial_{i}$ denotes the action of $\partial$ on the $i$-th factor in the tensor product $V^{\otimes n}$. Then, for every $\alpha=1, \ldots, s$,

$$
\frac{\partial}{\partial \lambda_{i}} f_{\lambda_{1}, \ldots, \lambda_{n}}^{\Gamma}\left(v_{1} \otimes \cdots \otimes v_{n}\right) \text { is the same for all } i \in I_{\alpha}
$$

and

$$
f_{\lambda_{1}, \ldots, \lambda_{n}}^{\Gamma}\left(\partial_{\Gamma_{\alpha}}\left(v_{1} \otimes \cdots \otimes v_{n}\right)\right)=-\lambda_{\Gamma_{\alpha}} f_{\lambda_{1}, \ldots, \lambda_{n}}^{\Gamma}\left(v_{1} \otimes \cdots \otimes v_{n}\right) .
$$

Observe that the second sesquilinearity condition (3.29) implies

$$
f_{\lambda_{1}, \ldots, \lambda_{n}}^{\Gamma}(\partial v)=-\sum_{i=1}^{n} \lambda_{i} f_{\lambda_{1}, \ldots, \lambda_{n}}^{\Gamma}(v)=\partial\left(f_{\lambda_{1}, \ldots, \lambda_{n}}^{\Gamma}(v)\right), \quad v \in V^{\otimes n} .
$$


Remark 3.8. When the graph $\Gamma$ is connected, the first sesquilinearity condition (3.28) implies that $f_{\lambda_{1}, \ldots, \lambda_{n}}^{\Gamma}\left(v_{1} \otimes \cdots \otimes v_{n}\right)$ is a polynomial of $\lambda_{1}+\cdots+\lambda_{n}$. Hence, it is an element of

$$
V\left[\lambda_{1}+\cdots+\lambda_{n}\right] /\left\langle\partial+\lambda_{1}+\cdots+\lambda_{n}\right\rangle \simeq V .
$$

In this case, we will omit the subscripts of $f^{\Gamma}$.

The classical operad $\mathcal{P}_{\mathrm{cl}}(V)$ is defined as the collection of the vector superspaces $\mathcal{P}_{\mathrm{cl}}(n), n \geq 0$, endowed, for every $f \in \mathcal{P}_{\mathrm{cl}}(n)$ and $m_{1}, \ldots, m_{n} \geq 0$, with the composition parity preserving linear map

$$
\begin{aligned}
\mathcal{P}_{\mathrm{cl}}(n) \otimes \mathcal{P}_{\mathrm{cl}}\left(m_{1}\right) \otimes \cdots \otimes \mathcal{P}_{\mathrm{cl}}\left(m_{n}\right) & \rightarrow \mathcal{P}_{\mathrm{cl}}\left(M_{n}\right), \\
f \otimes g_{1} \otimes \cdots \otimes g_{n} & \mapsto f\left(g_{1}, \ldots, g_{n}\right),
\end{aligned}
$$

described as follows. Let $M_{i}$ be as in (3.5), and

$$
\Lambda_{i}=\sum_{j=M_{i-1}+1}^{M_{i}} \lambda_{j}, \quad i=1, \ldots, n .
$$

If $\Gamma \in \mathcal{G}\left(M_{n}\right)$, then

$$
\left(f\left(g_{1}, \ldots, g_{n}\right)\right)^{\Gamma}: V^{\otimes M_{n}} \rightarrow V\left[\lambda_{1}, \ldots, \lambda_{M_{n}}\right] /\left\langle\partial+\lambda_{1}+\cdots+\lambda_{M_{n}}\right\rangle
$$

is defined by the formula:

$$
\begin{aligned}
& \left(f\left(g_{1}, \ldots, g_{n}\right)\right)_{\lambda_{1}, \ldots, \lambda_{M_{n}}}^{\Gamma}\left(v_{1} \otimes \cdots \otimes v_{M_{n}}\right) \\
& =f_{\Lambda_{1}, \ldots, \Lambda_{n}}^{\Delta_{0}^{m_{1} \ldots m_{n}}(\Gamma)}\left(\left(\left(\left.\right|_{x_{1}=\Lambda_{1}+\partial}\left(g_{1}\right)_{\lambda_{1}+X(1), \ldots, \lambda_{M_{1}}+X\left(M_{1}\right)}^{\Delta_{1}^{m_{1} \ldots m_{n}}(\Gamma)}\right) \otimes \cdots\right.\right. \\
& \left.\left.\cdots \otimes\left(\left.\right|_{x_{n}=\Lambda_{n}+\partial}\left(g_{n}\right)_{\lambda_{M_{n-1}+1}^{m_{1} \ldots m_{n}}(\Gamma)}^{\Delta_{m_{n}}+X\left(M_{n-1}+1\right), \ldots, \lambda_{M_{n}}+X\left(M_{n}\right)}\right)\right)\left(v_{1} \otimes \cdots \otimes v_{M_{n}}\right)\right)
\end{aligned}
$$

where $\Delta^{m_{1}, \ldots, m_{n}}(\Gamma)$ is the cocomposition of $\Gamma$ described in Section 3.3, $X(1), \ldots$ $\ldots, X\left(M_{n}\right)$ are the variables as in (3.10), and the notation is as follows. For given graphs $\Gamma_{1} \in \mathcal{G}\left(m_{1}\right), \ldots, \Gamma_{n} \in \mathcal{G}\left(m_{n}\right)$, we have:

$$
\begin{aligned}
& \left(\left(g_{1}\right)_{\lambda_{1}, \ldots, \lambda_{M_{1}}}^{\Gamma_{1}} \otimes \cdots \otimes\left(g_{n}\right)_{\lambda_{M_{n-1}+1}, \ldots, \lambda_{M_{n}}}^{\Gamma_{n}}\right)\left(v_{1} \otimes \cdots \otimes v_{M_{n}}\right) \\
& :=(-1)^{\sum_{i<j} p\left(g_{j}\right)\left(p\left(v_{M_{i-1}+1}\right)+\cdots+p\left(v_{M_{i}}\right)\right)}\left(g_{1}\right)_{\lambda_{1}, \ldots, \lambda_{M_{1}}}^{\Gamma_{1}}\left(v_{1} \otimes \cdots \otimes v_{M_{1}}\right) \otimes \cdots \\
& \cdots \otimes\left(g_{n}\right)_{\lambda_{M_{n-1}+1}, \ldots, \lambda_{M_{n}}}^{\Gamma_{n}}\left(v_{M_{n-1}+1} \otimes \cdots \otimes v_{M_{n}}\right),
\end{aligned}
$$

and for polynomials $P(\lambda)=\sum_{m} p_{m} \lambda^{m}$ and $Q(\mu)=\sum_{n} q_{n} \mu^{n}$ with coefficients in $V$, we write

$$
\left(\left.\right|_{x=\partial} P(\lambda+y)\right) \otimes\left(\left.\right|_{y=\partial} Q(\mu+x)\right)=\sum_{m, n}\left((\mu+\partial)^{n} p_{m}\right) \otimes\left((\lambda+\partial)^{m} q_{n}\right) .
$$

For each $n \geq 1, \mathcal{P}_{\mathrm{cl}}(n)$ has a natural right action of the symmetric group $S_{n}$, which is given by $\left(f \in \mathcal{P}_{\mathrm{cl}}(n), \Gamma \in \mathcal{G}(n), v_{1}, \ldots, v_{n} \in V\right)$ :

$$
\left(f^{\sigma}\right)_{\lambda_{1}, \ldots, \lambda_{n}}^{\Gamma}\left(v_{1} \otimes \cdots \otimes v_{n}\right)=f_{\sigma\left(\lambda_{1}, \ldots, \lambda_{n}\right)}^{\sigma(\Gamma)}\left(\sigma\left(v_{1} \otimes \cdots \otimes v_{n}\right)\right),
$$

where $\sigma\left(\lambda_{1}, \ldots, \lambda_{n}\right)$ is defined by (3.1), $\sigma\left(v_{1} \otimes \cdots \otimes v_{n}\right)$ is defined by (3.2), and $\sigma(\Gamma)$ is defined in Section 3.3. 
On the space $\mathcal{P}_{\mathrm{cl}}(n)$ we can also define a grading:

$$
\mathcal{P}_{\mathrm{cl}}(n)=\bigoplus_{r \geq 0} \operatorname{gr}^{r} \mathcal{P}_{\mathrm{cl}}(n),
$$

where $\operatorname{gr}^{r} \mathcal{P}_{\mathrm{cl}}(n)$ is the subspace of all maps in $\mathcal{P}_{\mathrm{cl}}(n)$ vanishing on graphs with a number of edges not equal to $r$. Then $\mathcal{P}_{\mathrm{cl}}$ is a graded operad, i.e., the compositions and the actions of the symmetric groups are compatible with the grading.

3.8. PVA cohomology [BDSHK18]. Given a vector superspace $V$ with parity $p$, and an even endomorphism $\partial \in \operatorname{End}(V)$, let $\Pi V$ be the same vector space with reversed parity $\bar{p}=1-p$, and consider the corresponding classical operad $\mathcal{P}_{\mathrm{cl}}(\Pi V)$ from Section 3.7. The associated $\mathbb{Z}$-graded Lie superalgebra is $W_{\mathrm{cl}}(\Pi V):=$ $W\left(\mathcal{P}_{\mathrm{cl}}(\Pi V)\right)$, with Lie bracket defined by (3.22).

Theorem 3.9 ([BDSHK18, Theorem 10.7]). We have a bijective correspondence between the odd elements $X \in W_{\mathrm{cl}}^{1}(\Pi V)$, such that $X \square X=0$, and the Poisson vertex superalgebra structures on $V$, defined as follows. The commutative associative product and the $\lambda$-bracket of the Poisson vertex superalgebra $V$ corresponding to $X$ are given by

$$
a b=(-1)^{p(a)} X^{\bullet-\bullet}(a \otimes b), \quad\left[a_{\lambda} b\right]=(-1)^{p(a)} X_{\lambda,-\lambda-\partial}^{\bullet}(a \otimes b) .
$$

Thanks to the Jacobi identity for the Lie superalgebra $W_{\mathrm{cl}}(\Pi V)$, if $X \in W_{\mathrm{cl}}^{1}(\Pi V)_{\overline{1}}$ satisfies $X \square X=0$, then $(\operatorname{ad} X)^{2}=0$. In view of Theorem 3.9, this means that we have a cohomology complex

$$
\left(W_{\mathrm{cl}}(\Pi V), \operatorname{ad} X\right),
$$

called the PVA cohomology complex, where $X \in W^{1}(\Pi V)_{\overline{1}}$ is given by (3.37).

\section{Relation between PVA and differential Harrison COHOMOlOGy} COMPLEXES

4.1. Main theorem. Let $V$ be a Poisson vertex algebra. By Theorem 3.9, we have an odd element $X \in W_{\mathrm{cl}}(\Pi V)$ such that $[X, X]=0$, which is associated to the PVA structure of $V$ by (3.37). Thus, there is the PVA cohomology complex

$$
\left(W_{\mathrm{cl}}(\Pi V), \operatorname{ad} X\right) \text {. }
$$

A classical $n$-cochain is an element $Y \in W_{\mathrm{cl}}^{n-1}(\Pi V)$, namely a map

$$
Y: \mathcal{G}(n) \times(\Pi V)^{\otimes n} \longrightarrow(\Pi V)\left[\lambda_{1}, \ldots, \lambda_{n}\right] /\left\langle\partial+\lambda_{1}+\cdots+\lambda_{n}\right\rangle,
$$

satisfying relations (3.25), (3.26), (3.28), (3.29), and the following symmetry property (by definition (3.20)):

$$
Y^{\sigma}=Y, \quad \forall \sigma \in S_{n} .
$$

Recall the grading of the superoperad $\mathcal{P}_{\mathrm{cl}}(\Pi V)$ from (3.36): $\operatorname{gr}^{r} W_{\mathrm{cl}}^{n-1}(\Pi V)$ is the set of maps $Y$ as in (4.2) such that

$$
Y^{\Gamma}=0 \text { unless }|E(\Gamma)|=r .
$$

Note that if $\Gamma \in \mathcal{G}(n)$ has $|E(\Gamma)| \geq n$, then necessarily $\Gamma$ contains a cycle. Hence, by the cycle relation (3.25), $Y^{\Gamma}=0$. Therefore the top degree in $\operatorname{gr} W_{\mathrm{cl}}^{n-1}(\Pi V)$ is $n-1$, i.e.

$$
\operatorname{gr}^{r} W_{\mathrm{cl}}^{n-1}(\Pi V)=0 \quad \text { if } r \geq n .
$$


Note that, if $\Gamma \in \mathcal{G}_{0}(n)$, then $|E(\Gamma)|=n-1$ if and only if $\Gamma$ is connected. By Remark 3.8, the top degree subspace $\mathrm{gr}^{n-1} W_{\mathrm{cl}}^{n-1}(\Pi V)$ consists of all collections of maps

$$
Y^{\Gamma}:(\Pi V)^{\otimes n} \longrightarrow(\Pi V), \quad \text { for } \Gamma \in \mathcal{G}_{0}(n), \quad|E(\Gamma)|=n-1,
$$

satisfying (3.25), (3.26), (4.3), and $Y^{\Gamma}\left(\partial\left(v_{1} \otimes \cdots \otimes v_{n}\right)\right)=\partial Y^{\Gamma}\left(v_{1} \otimes \cdots \otimes v_{n}\right)$. If $\Gamma$ is not connected, then $Y^{\Gamma}=0$.

In addition, as explained in Section 2.3, there is another cohomology complex associated to $V$, viewed as a differential algebra, namely the differential Harrison complex

$$
\left(C_{\partial, \mathrm{Har}}(V), d\right) \text {, }
$$

where $C_{\partial, \mathrm{Har}}^{n}(V) \subset \operatorname{Hom}_{\mathbb{F}[\partial]}\left(V^{\otimes n}, V\right)$ is defined by Harrison's conditions (2.11) and $d$ is the Hochschild differential (2.2).

The main result of this paper is the following:

Theorem 4.1. Let $V$ be a Poisson vertex algebra. One has a surjective morphism of cochain complexes

$$
\left(W_{\mathrm{cl}}(\Pi V), \operatorname{ad} X\right) \rightarrow\left(C_{\partial, \operatorname{Har}}(V), d\right),
$$

mapping $Y \in W_{\mathrm{cl}}^{n-1}(\Pi V)$ to $Y^{\Lambda_{n}}$, where $\Lambda_{n}$ is the standard n-line

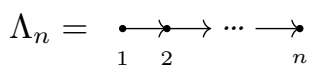

Moreover, the morphism (4.7) restricts to a bijective linear map on the top degree:

$$
\mathrm{gr}^{n-1} W_{\mathrm{cl}}^{n-1}(\Pi V) \stackrel{\sim}{\rightarrow} C_{\partial, \mathrm{Har}}^{n}(V) .
$$

We will prove Theorem 4.1 in Section 4.6. For that, we will need some preliminary results.

4.2. Lines. We say that a graph $\Gamma \in \mathcal{G}(n)$ is a non-connected line if it has the following form:

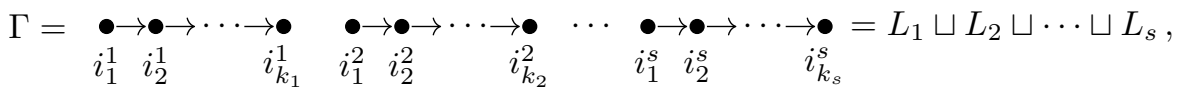

where $1 \leq k_{1} \leq \cdots \leq k_{s}$ are such that $k_{1}+\cdots+k_{s}=n$, and the set of indices $\left\{i_{b}^{a}\right\}$ is a permutation of $\{1, \ldots, n\}$ such that

$$
i_{1}^{l}=\min \left\{i_{1}^{l}, \ldots, i_{k_{l}}^{l}\right\} \quad \forall l=1, \ldots, s .
$$

If $k_{l}=k_{l+1}$, we also assume that $i_{1}^{l}<i_{1}^{l+1}$. In particular, the connected lines are all of the form

$$
\sigma\left(\Lambda_{n}\right), \quad \sigma \in S_{n} \text { such that } \sigma(1)=1,
$$

where $\Lambda_{n}$ is the $n$-line (4.8). Let $\mathcal{L}(n)$ be the set of $n$-graphs that are non-connected lines. Let also $\mathbb{F} \mathcal{G}(n)$ be the vector space with basis the set of graphs $\mathcal{G}(n)$.

Definition 4.2. The cycle relations in $\mathbb{F} \mathcal{G}(n)$ are the following elements:

(i) all $\Gamma \in \mathcal{G}(n) \backslash \mathcal{G}_{0}(n)$ (i.e., graphs containing a cycle);

(ii) all linear combinations $\sum_{e \in C} \Gamma \backslash e$, where $\Gamma \in \mathcal{G}(n)$ and $C \subset E(\Gamma)$ is an oriented cycle. 
Denote by $R(n) \subset \mathbb{F} \mathcal{G}(n)$ the subspace spanned by the cycle relations (i) and (ii).

Note that reversing an arrow in a graph $\Gamma \in \mathcal{G}(n)$ gives us, modulo cycle relations, the element $-\Gamma \in \mathbb{F} \mathcal{G}(n)$.

Example 4.3. For $n=3$, a cycle relation of type (ii) is:

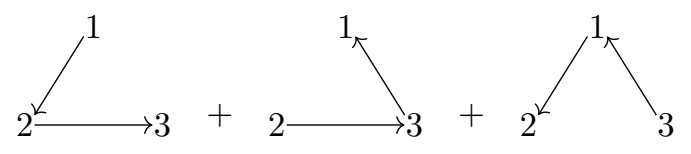

Remark 4.4. The cycle relations (3.25) and (3.26) on $Y \in \mathcal{P}_{\mathrm{cl}}$ can be restated by saying that $Y^{\Gamma}=0$ for all $\Gamma \in R(n)$.

Theorem 4.5 ([BDSHK19, Theorem 4.7]). The set $\mathcal{L}(n)$ is a basis for the quotient space $\mathbb{F} \mathcal{G}(n) / R(n)$.

By Theorem 4.5 and (4.12), we can write every connected graph $\Gamma \in \mathcal{G}(n)$, uniquely, modulo cycle relation, as follows:

$$
\Gamma \equiv \sum_{\substack{\sigma \in S_{n} \\ \sigma(1)=1}} c_{\sigma}^{\Gamma} \sigma \Lambda_{n},
$$

where $c_{\sigma}^{\Gamma} \in \mathbb{F}$ and the action of the symmetric group on graphs is defined in Section 3.3. Here and further, by $\equiv$ we mean equivalence modulo cycle relations, i.e., equality in the quotient space $\mathbb{F} \mathcal{G}(n) / R(n)$.

\subsection{Connected lines.}

Lemma 4.6. For every $n$, the following identity on $n$-lines holds:

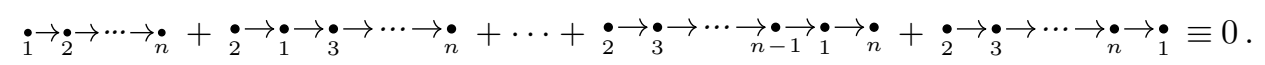

Proof. Let us consider the first two terms in the left-hand side of (4.15). Reversing the edges $2 \rightarrow 1$ and $1 \rightarrow 3$ in the second graph, and using (4.13), we have:

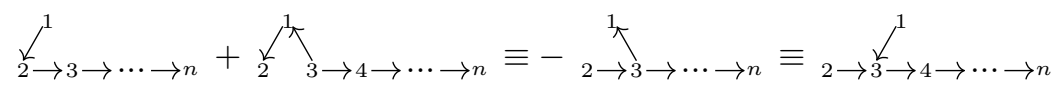

Adding (4.16) to the third term appearing in the left-hand side of (4.15), and applying again (4.13), we obtain:

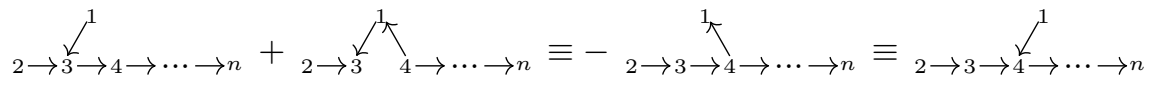

We proceed in the same way, up to

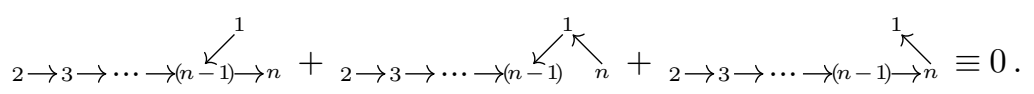

This completes the proof of the lemma. 
Remark 4.7. Observe that equation (4.15) can be viewed as a "local" identity: Lemma 4.6 holds even if we attach the same graph $\Gamma$ at any vertex of every graph appearing in the identity.

Lemma 4.8. Let $\Lambda_{n}$ be as in (4.8). For every $k \in\{2, \ldots, n\}$, the following identity holds:

$$
\Lambda_{n}+(-1)^{k} \sum_{\pi \in \mathcal{M}_{n}^{k}} \pi \Lambda_{n} \equiv 0
$$

where the sum is over all monotone permutations $\pi$ starting at $k$ and the action of $S_{n}$ on graphs is described in Section 3.3.

Proof. If $k=2$, then (4.19) is equivalent to (4.15). Suppose that $k>2$. Recall the description (2.5) of the monotone permutations $\pi \in \mathcal{M}_{n}^{k}$ in terms of the set $D(\pi)$ of drops. Given the set of drops $D=\left\{d_{1}, \ldots, d_{k-1}\right\}$ such that $2 \leq d_{k-1}<\cdots<d_{1} \leq$ $n$, the corresponding monotone permutation $\pi^{D} \in \mathcal{M}_{n}^{k}$ is uniquely determined by $\pi^{-1}(i)=d_{i}, \forall i=1 \ldots, k-1$. Hence:

$$
\pi^{D}\left(\Lambda_{n}\right)=k \rightarrow(k+1) \rightarrow \cdots \rightarrow \underset{\left[d_{k-1}\right]}{\rightarrow(k-1)} \rightarrow \cdots \rightarrow \underline{2} \rightarrow \cdots \rightarrow \underline{1} \rightarrow \cdots \rightarrow n
$$

where the underlined positions correspond to drops, while all other positions have vertices in increasing order from $k+1$ to $n$.

We then have:

$$
\sum_{\pi \in \mathcal{M}_{n}^{k}} \pi \Lambda_{n}=\sum_{2 \leq d_{k-1}<\cdots<d_{1} \leq n} k \rightarrow(k+1) \rightarrow \cdots \rightarrow \underset{\left[d_{k-1}\right]}{\rightarrow(k-1)} \rightarrow \underset{\left[d_{2}\right]}{\rightarrow \infty} \rightarrow \underset{\left[d_{1}\right]}{\rightarrow} \rightarrow \cdots \rightarrow n
$$

By Lemma 4.6, the sum over $d_{1} \in\left\{d_{2}+1, \ldots, n\right\}$ gives

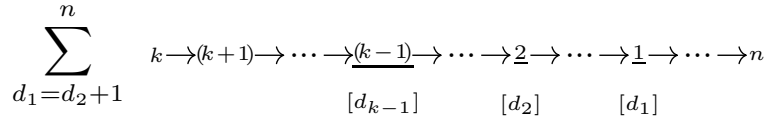

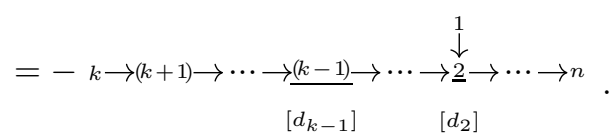

Similarly, using again Lemma 4.6 (cf. Remark 4.7), the sum over $d_{2} \in\left\{d_{3}+1, \ldots, n\right\}$ is

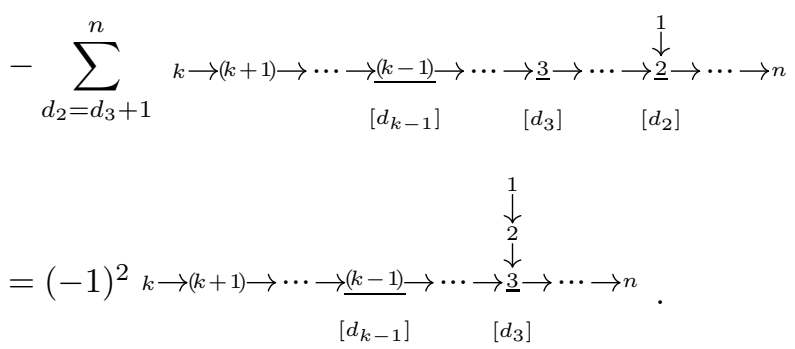


Repeating the same argument $k$ times, we conclude that

$$
\begin{aligned}
& \sum_{\pi \in \mathcal{M}_{n}^{k}} \pi \Lambda_{n}=(-1)^{k-2} \sum_{d_{k-1}=2}^{n} k \rightarrow(k+1) \rightarrow \cdots \rightarrow \underset{\substack{(k-1) \\
\left[d_{k-1}\right]}}{\downarrow} \rightarrow \cdots \rightarrow n
\end{aligned}
$$

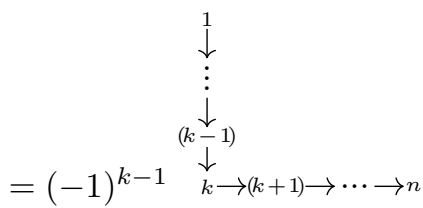

$$
\begin{aligned}
& =(-1)^{k-1} \Lambda_{n} \text {, }
\end{aligned}
$$

proving the lemma.

4.4. Relation between the symmetry property and Harrison's conditions. Recall that every $Y \in W_{\mathrm{cl}}^{n-1}(\Pi V)$ satisfies the symmetry property (4.3).

Lemma 4.9. If $Y \in W_{\mathrm{cl}}^{n-1}(\Pi V)$, then $Y^{\Lambda_{n}}$ satisfies Harrison's relations (2.11), hence it lies in the differential Harrison cohomology complex:

$$
Y^{\Lambda_{n}} \in C_{\partial, \mathrm{Har}}^{n}(V) .
$$

Conversely, given $F \in C_{\partial, \mathrm{Har}}^{n}(V)$, there exists a unique $Y \in \mathrm{gr}^{n-1} W_{\mathrm{cl}}^{n-1}(\Pi V)$, such that

$$
Y^{\Lambda_{n}}=F .
$$

Consequently, the linear map

$$
\mathrm{gr}^{n-1} W_{\mathrm{cl}}^{n-1}(\Pi V) \stackrel{\sim}{\rightarrow} C_{\partial, \mathrm{Har}}^{n}(V), \quad Y \mapsto Y^{\Lambda_{n}}
$$

is bijective.

Proof. First, we prove that, if $Y \in W_{\mathrm{cl}}^{n-1}(\Pi V)$ satisfies the symmetry relations (4.3), then $f=Y^{\Lambda_{n}}$ satisfies Harrison's conditions (2.11). By Lemma 4.8 (cf. Remark 4.4), we get

$$
Y^{\Lambda_{n}}=(-1)^{k-1} \sum_{\pi \in \mathcal{M}_{n}^{k}} Y^{\pi\left(\Lambda_{n}\right)}
$$

Evaluating both sides of this identity on $v_{1} \otimes \cdots \otimes v_{n} \in V^{\otimes n}$, the left side is simply $Y^{\Lambda_{n}}\left(v_{1} \otimes \cdots \otimes v_{n}\right)=f\left(v_{1} \otimes \cdots \otimes v_{n}\right)$. On the right-hand side, we have

$$
\begin{gathered}
(-1)^{k-1} \sum_{\pi \in \mathcal{M}_{n}^{k}} Y^{\pi\left(\Lambda_{n}\right)}\left(v_{1} \otimes \cdots \otimes v_{n}\right)=(-1)^{k-1} \sum_{\pi \in \mathcal{M}_{n}^{k}}\left(Y^{\pi^{-1}}\right)^{\pi\left(\Lambda_{n}\right)}\left(v_{1} \otimes \cdots \otimes v_{n}\right) \\
=(-1)^{k-1} \sum_{\pi \in \mathcal{M}_{n}^{k}} \operatorname{sign}(\pi) Y^{\Lambda_{n}}\left(v_{\pi(1)} \otimes \cdots \otimes v_{\pi(n)}\right) \\
=L_{k} f\left(v_{1} \otimes \cdots \otimes v_{n}\right),
\end{gathered}
$$

by the definition (2.10) of $L_{k}$. Hence, $f$ satisfies Harrison's conditions (2.11) as claimed.

We next turn to the second claim of the lemma. Let $F \in C_{\partial \text {,Har }}^{n}(V)$, i.e., $F: V^{\otimes n} \rightarrow V$ is an $\mathbb{F}[\partial]$-module homomorphism satisfying Harrison's conditions 
(2.11). Then the corresponding $Y \in \operatorname{gr}^{n-1} W_{\mathrm{cl}}^{n-1}(\Pi V)$, such that $Y^{\Lambda_{n}}=F$, is defined as follows. For $\Gamma \in R(n)$, or if $\Gamma \in \mathcal{L}(n)$ is not connected, we set

$$
Y^{\Gamma}=0 \text {. }
$$

For $\Gamma \in \mathcal{L}(n)$ connected, there exists a unique $\tau \in S_{n}$ such that $\tau(1)=1$ and $\Gamma=\tau\left(\Lambda_{n}\right)$. We then set

$$
Y^{\Gamma}\left(v_{1} \otimes \cdots \otimes v_{n}\right)=\operatorname{sign}(\tau) F\left(v_{\tau(1)} \otimes \cdots \otimes v_{\tau(n)}\right) .
$$

In particular, for $\Gamma=\Lambda_{n}$, we have $\tau=1$ and $Y^{\Lambda_{n}}=F$.

Since, by Theorem $4.5, \mathcal{L}(n)$ is a basis for the vector space $\mathbb{F} \mathcal{G}(n) / R(n)$, equations (4.20) and (4.21) determine a unique well-defined element $Y \in \mathrm{gr}^{n-1} \mathcal{P}_{\mathrm{cl}}(\Pi V)(n)$. Indeed, $Y$ satisfies the cycle relations (3.25) and (3.26), by (4.20), Theorem 4.5 and Remark 4.4. The first sesquilinearity condition (3.28) is obvious, and the second condition (3.29) follows from (3.30).

To prove that $Y \in \mathrm{gr}^{n-1} W_{\mathrm{cl}}^{n-1}(\Pi V)$, it remains to show that $Y$ satisfies the symmetry conditions (4.3). Obviously, the action of the symmetric group $S_{n}$ preserves $R(n)$ and the set of non-connected lines. Hence, when we evaluate (4.3) on $\Gamma \in R(n)$ or on a non-connected $n$-line $\Gamma \in \mathcal{L}(n)$, we get $0=0$.

We are left to prove that (4.3) holds when evaluated on a connected line $\Gamma \in \mathcal{L}(n)$, which, as remarked above, can be obtained as $\Gamma=\tau\left(\Lambda_{n}\right)$, for a unique $\tau \in S_{n}$ such that $\tau(1)=1$. The right-hand side of (4.3), when evaluated on such a $\Gamma$ is given by (4.21). The left-hand side is, by (3.35),

$$
\begin{aligned}
\left(Y^{\sigma}\right)^{\Gamma}\left(v_{1} \otimes \cdots \otimes v_{n}\right) & =Y^{\sigma \tau\left(\Lambda_{n}\right)}\left(\sigma\left(v_{1} \otimes \cdots \otimes v_{n}\right)\right) \\
& =\operatorname{sign}(\sigma) Y^{\sigma \tau\left(\Lambda_{n}\right)}\left(v_{\sigma(1)} \otimes \cdots \otimes v_{\sigma_{n}}\right) .
\end{aligned}
$$

By Lemma 4.8, we have, modulo $R(n)$,

$$
\sigma \tau\left(\Lambda_{n}\right) \equiv(-1)^{\tau^{-1} \sigma^{-1}(1)-1} \sum_{\pi \in \mathcal{M}_{n}^{\tau^{-1} \sigma^{-1}(1)}} \sigma \tau \pi\left(\Lambda_{n}\right) .
$$

Hence, by Remark 4.4, the right-hand side of (4.22) becomes

$$
\operatorname{sign}(\sigma)(-1)^{\tau^{-1} \sigma^{-1}(1)-1} \sum_{\pi \in \mathcal{M}_{n}^{\tau^{-1} \sigma^{-1}(1)}} Y^{\sigma \tau \pi\left(\Lambda_{n}\right)}\left(v_{\sigma^{-1}(1)} \otimes \cdots \otimes v_{\sigma^{-1}(n)}\right) .
$$

Note that, if $\pi \in \mathcal{M}_{n}^{\tau^{-1} \sigma^{-1}(1)}$, then $\sigma \tau \pi(1)=1$. Hence, we can apply formula (4.21) to get

$$
Y^{\sigma \tau \pi\left(\Lambda_{n}\right)}\left(v_{\sigma^{-1}(1)} \otimes \cdots \otimes v_{\sigma^{-1}(n)}\right)=\operatorname{sign}(\sigma \tau \pi) F\left(v_{\tau \pi(1)} \otimes \cdots \otimes v_{\tau \pi(n)}\right) .
$$

Combining (4.22)-(4.24), we get, by (2.7) and the definition (2.10) of $L_{k}$,

$$
\begin{aligned}
& \left(Y^{\sigma}\right)^{\Gamma}\left(v_{1} \otimes \cdots \otimes v_{n}\right) \\
& =\operatorname{sign}(\tau)(-1)^{\tau^{-1} \sigma^{-1}(1)-1} \sum_{\pi \in \mathcal{M}_{n}^{\tau^{-1} \sigma^{-1}(1)}} \operatorname{sign}(\pi) F\left(v_{\tau \pi(1)} \otimes \cdots \otimes v_{\tau \pi(n)}\right) \\
& =\operatorname{sign}(\tau)\left(L_{\tau^{-1} \sigma^{-1}(1)} F\right)\left(v_{\tau(1)} \otimes \cdots \otimes v_{\tau}(n)\right),
\end{aligned}
$$

which equals (4.21) by Harrison's conditions (2.11).

Hence, $Y$ is a well-defined element of $\operatorname{gr}^{n-1} W_{\mathrm{cl}}^{n-1}(\Pi V)$, such that $Y^{\Lambda_{n}}=F$, as required. The uniqueness of such a $Y$ is obvious since, by Theorem 4.5, $Y \in$ $\mathrm{gr}^{n-1} W_{\mathrm{cl}}^{n-1}(\Pi V)$ is uniquely determined by its value on $\Lambda_{n}$. 


\subsection{Relation between ad $X$ and the Hochschild differential.}

Lemma 4.10. For $Y \in W_{\mathrm{cl}}^{n-1}(\Pi V)$ and $X \in W_{\mathrm{cl}}^{1}(\Pi V)$ defined in (3.37), we have

$$
[X, Y]^{\Lambda_{n+1}}\left(v_{1} \otimes \cdots \otimes v_{n+1}\right)=(-1)^{n+1} d\left(Y^{\Lambda_{n}}\right)\left(v_{1} \otimes \cdots \otimes v_{n+1}\right),
$$

where $\Lambda_{n}$ is as in (4.8) and $d$ is the Hochschild differential (2.2).

Proof. Recall that, by definition (3.22), the adjoint action of $X$ on $Y$ is given by:

$$
\begin{aligned}
{[X, Y] } & =X \square Y-(-1)^{n-1} Y \square X \\
& =\sum_{\sigma \in S_{n, 1}}\left(X \circ_{1} Y\right)^{\sigma^{-1}}+(-1)^{n} \sum_{\tau \in S_{2, n-1}}\left(Y \circ_{1} X\right)^{\tau^{-1}},
\end{aligned}
$$

since $\bar{p}(X)=1$ and $\bar{p}(Y)=n-1$. The elements of $S_{n, 1}$ are

$$
\begin{aligned}
\sigma_{k} & =\left(\begin{array}{cccccccc|c}
1 & 2 & \cdots & k-1 & k & k+1 & \cdots & n & n+1 \\
1 & 2 & \cdots & k-1 & k+1 & k+2 & \cdots & n+1 & k
\end{array}\right) \\
& =\left(\begin{array}{lllll}
k & k+1 & \cdots & n+1
\end{array}\right)
\end{aligned}
$$

for $1 \leq k \leq n+1$. The elements of $S_{2, n-1}$ are

$$
\tau_{i, j}=\left(\begin{array}{cc|ccccccccc}
1 & 2 & 3 & \cdots & i+1 & i+2 & \cdots & j & j+1 & \cdots & n+1 \\
i & j & 1 & \cdots & i-1 & i+1 & \cdots & j-1 & j+1 & \cdots & n+1
\end{array}\right),
$$

for $1 \leq i<j-1 \leq n$, and

$$
\tau_{i, i+1}=\left(\begin{array}{cc|cccccc}
1 & 2 & 3 & \cdots & i+1 & i+2 & \cdots & n+1 \\
i & i+1 & 1 & \cdots & i-1 & i+2 & \cdots & n+1
\end{array}\right)
$$

for $1 \leq i \leq n$.

Using (4.26), we evaluate

$$
\begin{aligned}
& \left(\left(X \circ_{1} Y\right)^{\sigma_{k}^{-1}}\right)^{\Lambda_{n+1}}\left(v_{1} \otimes \cdots \otimes v_{n+1}\right) \\
& \quad=(-1)^{n+1-k}\left(X \circ_{1} Y\right)^{\sigma_{k}^{-1}\left(\Lambda_{n+1}\right)}\left(v_{1} \otimes \cdots \otimes v_{k-1} \otimes v_{k+1} \otimes \cdots \otimes v_{n+1} \otimes v_{k}\right) .
\end{aligned}
$$

For $k=1$, by the symmetric group's action described in Section 3.3, we have

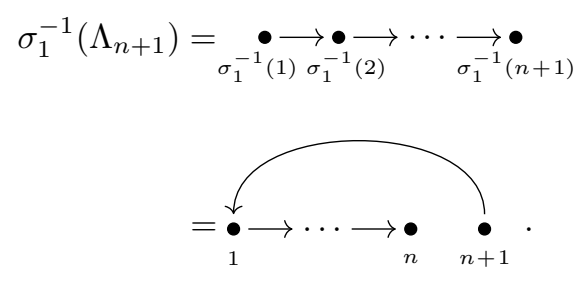

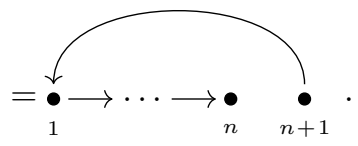

When we apply the cocomposition map $\Delta^{n, 1}$ to this graph, we get

$$
\Delta_{0}^{n, 1}\left(\sigma_{1}^{-1}\left(\Lambda_{n+1}\right)\right)=\underset{1}{\bullet} \longleftarrow
$$

and

$$
\Delta_{1}^{n, 1}\left(\sigma_{1}^{-1}\left(\Lambda_{n+1}\right)\right)=\underset{1}{\bullet} \underset{2}{\longrightarrow} \longrightarrow \cdots \underset{n}{\longrightarrow} \boldsymbol{\bullet}=\Lambda_{n}
$$


Hence, by the definition (3.32) of the composition map, (4.29) becomes

$$
\begin{aligned}
& \left(\left(X \circ_{1} Y\right)^{\sigma_{1}^{-1}}\right)^{\Lambda_{n+1}}\left(v_{1} \otimes \cdots \otimes v_{n+1}\right) \\
& \quad=(-1)^{n}\left(X \circ_{1} Y\right)^{\sigma_{1}^{-1}\left(\Lambda_{n+1}\right)}\left(v_{2} \otimes \cdots \otimes v_{n+1} \otimes v_{1}\right) \\
& \quad=(-1)^{n} X^{\bullet \leftarrow \bullet}\left(Y^{\Lambda_{n}}\left(v_{2} \otimes \cdots \otimes v_{n+1}\right) \otimes v_{1}\right) \\
& \quad=(-1)^{n+1} Y^{\Lambda_{n}}\left(v_{2} \otimes \cdots \otimes v_{n+1}\right) v_{1} .
\end{aligned}
$$

Similarly, for $k=n+1$, we have $\sigma_{n+1}=1$, and applying the cocomposition map $\Delta^{n, 1}$ to $\sigma_{n+1}^{-1}\left(\Lambda_{n+1}\right)=\Lambda_{n+1}$, we get:

$$
\Delta_{0}^{n, 1}\left(\sigma_{n+1}^{-1}\left(\Lambda_{n+1}\right)\right)=\stackrel{\bullet}{\longrightarrow} \underset{2}{\bullet}
$$

and

$$
\Delta_{1}^{n, 1}\left(\sigma_{n+1}^{-1}\left(\Lambda_{n+1}\right)\right)=\underset{1}{\bullet} \underset{2}{\longrightarrow} \longrightarrow \cdots \underset{n}{\longrightarrow}=\Lambda_{n}
$$

Then (4.29) becomes

$$
\begin{gathered}
\left(\left(X \circ_{1} Y\right)^{\sigma_{n+1}^{-1}}\right)^{\Lambda_{n+1}}\left(v_{1} \otimes \cdots \otimes v_{n+1}\right) \\
\quad=X^{\bullet \rightarrow \bullet}\left(Y^{\Lambda_{n}}\left(v_{1} \otimes \cdots \otimes v_{n}\right) \otimes v_{n+1}\right) \\
=Y^{\Lambda_{n}}\left(v_{1} \otimes \cdots \otimes v_{n}\right) v_{n+1} .
\end{gathered}
$$

Furthermore, for $2 \leq k \leq n$, we have

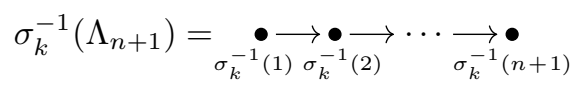

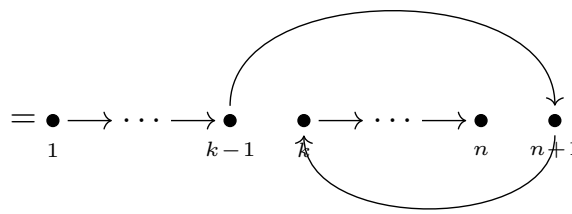

Hence, applying the cocomposition map $\Delta^{n, 1}$ we get

$$
\Delta_{0}^{n, 1}\left(\sigma_{k}^{-1}\left(\Lambda_{n+1}\right)\right)=\overbrace{2}
$$

which has a cycle. Therefore,

$$
\left(\left(X \circ_{1} Y\right)^{\sigma_{k}^{-1}}\right)^{\Lambda_{n+1}}=\overbrace{}^{\stackrel{\imath}{\mho}}(\ldots)=0 .
$$


Next, we compute $\left(\left(Y \circ_{1} X\right)^{\tau_{i, j}^{-1}}\right)^{\Lambda_{n+1}}\left(v_{1} \otimes \cdots \otimes v_{n+1}\right)$ where $\tau_{i, j}$ is defined in (4.27). By the symmetric group's action described in Section 3.3 we have

$$
\tau_{i, j}^{-1}\left(\Lambda_{n+1}\right)=\underset{\tau_{i, j}^{-1}(1) \tau_{i, j}^{-1}(2)}{\bullet} \cdots \underset{\tau_{i, j}^{-1}(n+1)}{\longrightarrow} \bullet \bullet
$$

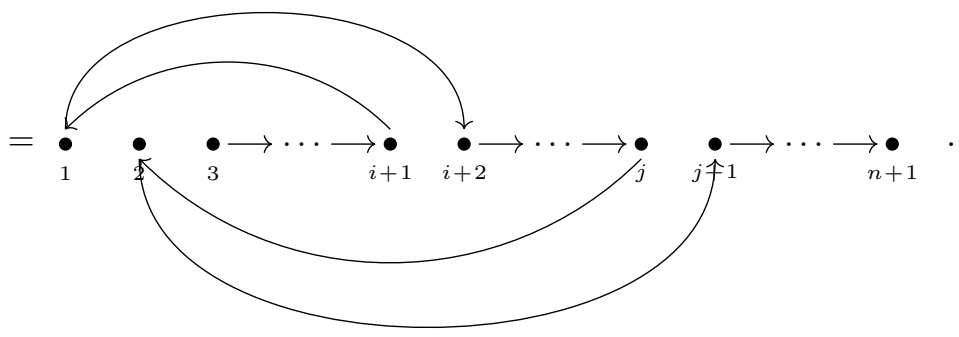

Hence, applying the cocomposition $\Delta^{2,1, \ldots, 1}$ we get

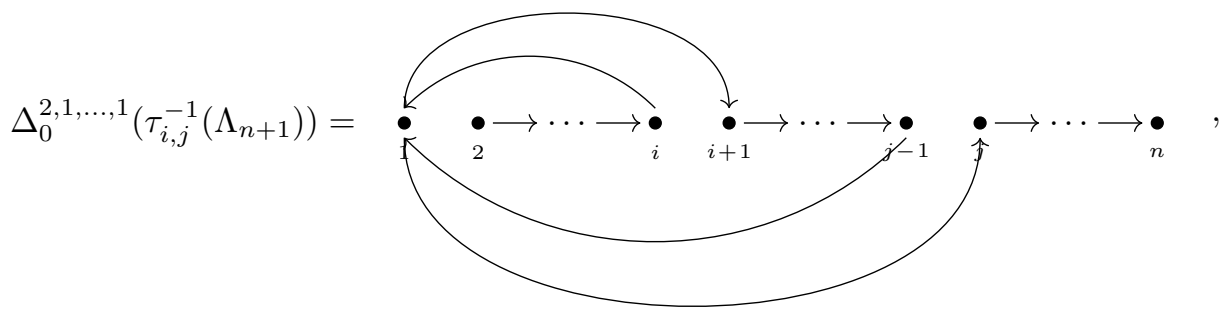

which has a cycle. Therefore,

$$
\left(\left(Y \circ_{1} X\right)^{\tau_{i, j}^{-1}}\right)^{\Lambda_{n+1}}=Y^{\Delta_{0}^{2,1, \ldots, 1}\left(\tau_{i, j}^{-1}\left(\Lambda_{n+1}\right)\right)}(\cdots)=0 .
$$

Finally, we evaluate $\left(\left(Y \circ_{1} X\right)^{\tau_{i, i+1}^{-1}}\right)^{\Lambda_{n+1}}\left(v_{1} \otimes \cdots \otimes v_{n+1}\right)$ with $\tau_{i, i+1}$ defined in (4.28). In this case, we have

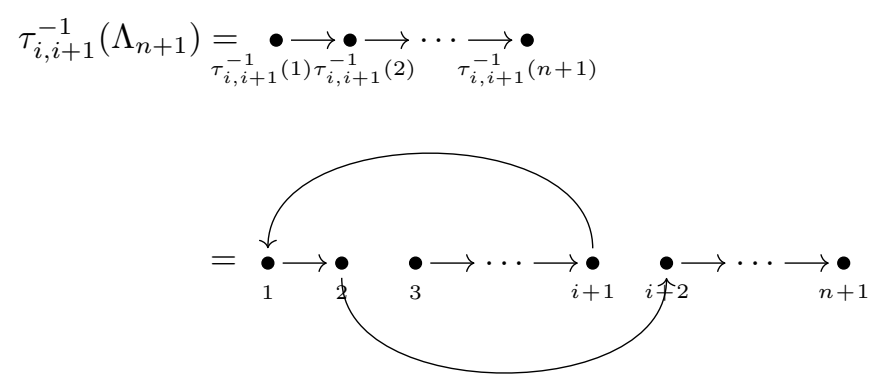


Hence, applying the cocomposition $\Delta^{2,1, \ldots, 1}$ we get

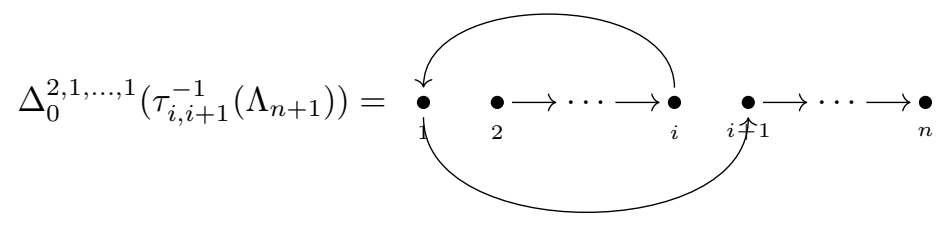

and

$$
\Delta_{1}^{2,1, \ldots, 1}\left(\tau_{i, i+1}^{-1}\left(\Lambda_{n+1}\right)\right)=\underset{1}{\bullet} \longrightarrow \bullet
$$

Therefore, by definition (3.32) of the composition map, we obtain

$$
\begin{aligned}
& \left(\left(Y \circ_{1} X\right)^{\tau_{i, j}^{-1}}\right)^{\Lambda_{n+1}}\left(v_{1} \otimes \cdots \otimes v_{n}\right)= \\
& =\left(Y \circ_{1} X\right)^{\tau_{i, j}^{-1}\left(\Lambda_{n+1}\right)}\left(v_{i} \otimes v_{i+1} \otimes v_{1} \otimes \cdots \otimes v_{i-1} \otimes v_{i+2} \otimes \cdots \otimes v_{n+1}\right) \\
& =Y^{\Delta_{0}^{2,1, \ldots, 1}\left(\tau_{i, i+1}^{-1}\left(\Lambda_{n+1}\right)\right)}\left(X^{\bullet-\bullet}\left(v_{i} \otimes v_{i+1}\right) \otimes v_{1} \otimes \cdots \otimes v_{i-1} \otimes v_{i+2} \otimes \cdots \otimes v_{n+1}\right) \\
& =Y^{\Delta_{0}^{2,1, \ldots, 1}\left(\tau_{i, i+1}^{-1}\left(\Lambda_{n+1}\right)\right)}\left(v_{i} v_{i+1} \otimes v_{1} \otimes \cdots \otimes v_{i-1} \otimes v_{i+2} \otimes \cdots \otimes v_{n+1}\right)
\end{aligned}
$$

Note that

$$
\Delta_{0}^{2,1, \ldots, 1}\left(\tau_{i, i+1}^{-1}\left(\Lambda_{n+1}\right)\right)=\sigma\left(\Lambda_{n}\right),
$$

where $\sigma=(12 \cdots i) \in S_{n}$ is the $i$-cycle. Hence, by the symmetry property (4.3), we can replace $Y$ by $Y^{\sigma^{-1}}$ in the right-hand side of (4.34) to get

$$
(-1)^{i+1} Y^{\Lambda_{n}}\left(v_{1} \otimes \cdots \otimes v_{i-1} \otimes v_{i} v_{i+1} \otimes v_{i+2} \otimes \cdots \otimes v_{n+1}\right) .
$$

Combining equations (4.25) and (4.30)-(4.35), we conclude that

$$
\begin{aligned}
& {[X, Y]^{\Lambda_{n+1}}\left(v_{1} \otimes \cdots \otimes v_{n}\right)} \\
& \quad=(-1)^{n+1} v_{1} Y^{\Lambda_{n}}\left(v_{2} \otimes \cdots \otimes v_{n+1}\right)+Y^{\Lambda_{n}}\left(v_{1} \otimes \cdots \otimes v_{n}\right) v_{n+1} \\
& \quad+(-1)^{n} \sum_{i=1}^{n}(-1)^{i+1} Y^{\Lambda_{n}}\left(v_{1} \otimes \cdots \otimes v_{i-1} \otimes v_{i} v_{i+1} \otimes v_{i+2} \otimes \cdots \otimes v_{n+1}\right) \\
& \quad=(-1)^{n+1} d\left(Y^{\Lambda_{n}}\right)\left(v_{1} \otimes \cdots \otimes v_{n}\right),
\end{aligned}
$$

completing the proof.

Corollary 4.11. Let $X$ be defined in (3.37) and $Y \in W_{\mathrm{cl}}^{n-1}(\Pi V)$ be such that $[X, Y]=0$. Then $Y^{\Lambda_{n}}$ is a cocyle in the Hochschild cohomology.

Proof. Obvious, by Lemma 4.10.

4.6. Proof of Theorem 4.1. By Lemma 4.9, given $Y \in W_{\mathrm{cl}}^{n-1}(\Pi V), Y^{\Lambda_{n}}$ is a cochain in the differential Harrison complex. Conversely, for any $F \in C_{\partial, \mathrm{Har}}^{n}(V)$, there is a unique $Y \in \mathrm{gr}^{n-1} W_{\mathrm{cl}}^{n-1}(\Pi V)$ such that $F=Y^{\Lambda_{n}}$. Hence, the following diagram is well defined:

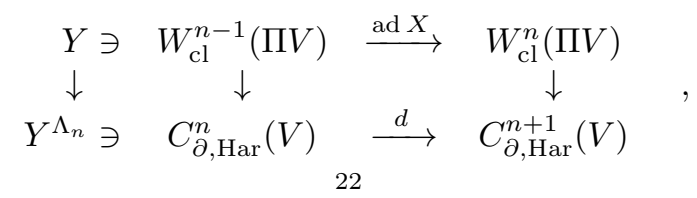


where the vertical maps are surjective and restrict to bijective maps on top degree: $\mathrm{gr}^{n-1} W_{\mathrm{cl}}^{n-1}(\Pi V) \stackrel{\sim}{\rightarrow} C_{\partial \text {,Har }}^{n}(V)$. Lemma 4.10 says that, up to a sign, the diagram (4.36) is commutative. This completes the proof of the theorem.

\section{REFERENCES}

[BDSHK18] B. Bakalov, A. De Sole, R. Heluani and V.G. Kac, An operadic approach to vertex algebra and Poisson vertex algebra cohomology. Japan. J. Math. 14 (2019), 1-94.

[BDSHK19] B. Bakalov, A. De Sole, R. Heluani and V.G. Kac, Chiral vs classical operad. Preprint arXiv:1812.05972.

[BDSHKV19] B. Bakalov, A. De Sole, R. Heluani, V.G. Kac, and V. Vignoli, Classical and variational Poisson cohomology, in preparation.

[BDSK19] B. Bakalov, A. De Sole, and V.G. Kac, Computation of cohomology of Lie conformal and Poisson vertex algebras. Preprint arXiv:1903.12059.

[DSK13] A. De Sole and V.G. Kac, Variational Poisson cohomology. Japan. J. Math. 8 (2013), $1-145$.

[GS87] M. Gerstenhaber and S.D. Schack, A Hodge-type decomposition for commutative algebra cohomology. J. Pure Appl. Algebra 48 (1987), 229-247.

[Har62] D.K. Harrison, Commutative algebras and cohomology. Trans. Amer. Math. Soc. 104 (1962), 191-204.

[Hoc45] G. Hochschild, On the cohomology groups of an associative algebra. Ann. of Math. (2) 46 (1945), 58-67.

[Lod13] J.L. Loday, Cyclic homology. Springer Science \& Business Media. vol. 301, 2013.

[Vig19] V. Vignoli, On Poisson vertex algebra cohomology. Ph.D. thesis, University of Rome La Sapienza, 2019.

Department of Mathematics, North Carolina State University, Raleigh, NC 27695, USA

E-mail address: bojko_bakalov@ncsu.edu

Dipartimento di Matematica, Sapienza Università di Roma, P.le Aldo Moro 2, 00185 Rome, Italy

E-mail address: desole@mat.uniroma1.it

$U R L$ : www1.mat.uniroma1.it/ desole

Department of Mathematics, Mit, 77 Massachusetts Ave., Cambridge, MA 02139, USA

E-mail address: kac@math.mit.edu

Dipartimento di Matematica, Sapienza Università di Roma, P.le Aldo Moro 2, 00185 Rome, Italy

E-mail address: vignoli@mat.uniroma1.it 